\title{
$\begin{array}{ll}\text { Research Square } & \text { Preprints are preliminary reports that have not undergone peer review. } \\ \text { They should not be considered conclusive, used to inform clinical practice, }\end{array}$ or referenced by the media as validated information.
}

\section{The Zariski topology on the graded second spectrum of a graded module}

\section{Saif Salam}

Jordan University of Science and Technology

Khaldoun Al-Zoubi ( $\nabla$ kfzoubi@just.edu.jo )

Jordan University of Science and Technology https://orcid.org/0000-0001-6082-4480

\section{Research Article}

Keywords: graded second submodules, graded second spectrum, graded prime submodules, Zariski topology

Posted Date: February 7th, 2022

DOI: https://doi.org/10.21203/rs.3.rs-1335019/v1

License: (1) This work is licensed under a Creative Commons Attribution 4.0 International License. Read Full License 


\title{
THE ZARISKI TOPOLOGY ON THE GRADED SECOND SPECTRUM OF A GRADED MODULE
}

\author{
SAIF SALAM AND KHALDOUN AL-ZOUBI*
}

\begin{abstract}
Let $R$ be a $G$-graded ring and $M$ be a $G$-graded $R$-module. The graded second spectrum of $M$, denoted by $\operatorname{Spec}_{G}^{s}(M)$, is the set of all graded second submodules of $M$. In this paper, we define a topology on $\operatorname{Spec}_{G}^{s}(M)$ which is analogous to that for $\operatorname{Spec}_{G}(R)$, and investigate several topological properties of this topology.
\end{abstract}

\section{Introduction AND Preliminaries}

Let $G$ be a multiplicative group with identity $e$ and $R$ be a commutative ring with identity 1 . Then $R$ is called a $G$-graded ring if there exist additive subgroups $R_{g}$ of $R$ indexed by the elements $g \in G$ such that $R=\underset{g \in G}{\bigoplus} R_{g}$ and $R_{g} R_{h} \subseteq R_{g h}$ for all $g, h \in G$. The elements of $R_{g}$ are called homogeneous elements of degree $g$. The homogeneous elements of the ring $R$ are denoted by $h(R)$, i.e. $h(R)=\bigcup_{g \in G} R_{g}$. In particular, $R_{e}$ is a subring of $R$ and $1 \in R_{e}$. If $x \in R$, then $x$ can be written uniquely as $\sum_{g \in G} x_{g}$, where $x_{g}$ is the component of $x$ in $R_{g}$. Let $R$ be a $G$-graded ring and $I$ be an ideal of $R$. Then $I$ is called a $G$-graded ideal of $R$, denoted by $I \triangleleft_{G} R$, if $I=\underset{g \in G}{\oplus}\left(I \cap R_{g}\right)$. A subring $H$ of $R$ is said to be graded subring if $H=\underset{g \in G}{\oplus}\left(H \cap R_{g}\right)$ (see $[9$ ). A proper graded ideal $J$ of $R$ is said to be graded maximal if there is no graded ideal $K$ of $R$ such that $J \varsubsetneqq K \varsubsetneqq R$. We will denote the set of all graded maximal ideals of $R$ by $\operatorname{Max}_{G}(R)$. The graded prime spectrum of $R$ is the set of all graded prime ideals of $R$ and it is denoted by $\operatorname{Spec}_{G}(R)$. It has been proved that $\operatorname{Spec}_{G}(R)$ is a topological space whose closed sets are $V_{G}^{R}(I)=\left\{p \in \operatorname{Spec}_{G}(R) \mid I \subseteq p\right\}$ for each graded ideal $I$ of $R$ (see, for example, $10-12]$ ).

Let $R$ be a $G$-graded ring and $M$ be a left $R$-module. Then $M$ is said to be a $G$-graded $R$-module if $M=\underset{g \in G}{\oplus} M_{g}$ with $R_{g} M_{h} \subseteq M_{g h}$ for all $g, h \in G$, where $M_{g}$ is an additive subgroup of $M$ for all $g \in G$. The elements of $M_{g}$ are called homogeneous of degree $g$. The set of all homogeneous elements

2010 Mathematics Subject Classification. 13A02, 16W50.

Key words and phrases. graded second submodules, graded second spectrum, graded prime submodules, Zariski topology.

* Corresponding author. 
of $M$ is denoted by $h(M)$, i.e. $h(M)=\bigcup_{g \in G} M_{g}$. If $m \in M$, then $m$ can be written uniquely as $\sum_{g \in G} m_{g}$, where $m_{g}$ is the component of $m$ in $M_{g}$. Let $M=\underset{g \in G}{\oplus} M_{g}$ be a $G$-graded $R$-module and $N$ be a submodule of $M$. For $g \in G$, let $N_{g}=N \cap M_{g}$. Then $N$ is called a $G$-graded submodule of $M$, denoted by $N \leq_{G} M$, if $N=\underset{g \in G}{\oplus_{g}} N_{g}$. In this case, $N_{g}$ is called the $g$-component of $N$ for $g \in G$. Also, $M / N$ becomes $G$-graded $R$-module with $g$-component $(M / N)_{g}=\left(M_{g}+N\right) / N$ for $g \in G$. Moreover, $M_{g}$ is an $R_{e}$-module and $N_{g}$ is an $R_{e}$-submodule of $M_{g}$ for $g \in G$, see 9 .

Let $M$ be a $G$-graded $R$-module. Let $I$ be a $G$-graded ideal of $R$ and $N$ be a $G$-graded submodule of $M$. Then $A n n_{R}(N)=\{r \in R \mid r N=\{0\}\}$ is a $G$-graded ideal of $R$. In addition, $\operatorname{Ann}_{M}(I)=\{m \in M \mid \operatorname{Im}=\{0\}\}$ and $\left(N:_{M} I\right)=\{m \in M \mid I m \subseteq N\}$ are $G$-graded submodules of $M$. In particular, $A n n_{M}(I)$ becomes a $G$-graded $R / I$-module by $\left(A n n_{M}(I)\right)_{g}=$ $A n n_{M}(I) \cap M_{g}$ for all $g \in G$.

A $G$-graded $R$-module $M$ is called a comultiplication graded $R$-module if every $N \leq_{G} M$ has the form $A n n_{M}(I)$ for some $I \triangleleft_{G} R$ (see, for example, [2,6]). It is clear that if $N$ is a graded submodule of a comultiplication graded module $M$, then $N=A n n_{M}\left(A n n_{R}(N)\right)$. A $G$-graded $R$-module $M$ is said to be graded Artinian if it satisfies the descending chain condition for graded submodules, see $[9]$.

Let $M$ and $M^{\prime}$ be graded $R$-modules. An $R$-module homomorphism $f$ : $M \rightarrow M^{\prime}$ is called graded homomorphism if $f\left(M_{g}\right) \subseteq M_{g}^{\prime}$ for all $g \in G$ (see [9]). A non-zero graded submodule $S$ of $M$ is said to be graded second if for each $r \in h(R)$, the graded $R$-homomorphism $f: S \rightarrow S$ defined by $f(x)=r x$ is either surjective or zero. In other words, $S$ is a graded second submodule of $M$ if $r S=S$ or $r S=0$ for every $r \in h(R)$. This implies that $p=A n n_{R}(S)$ is a graded prime ideal of $R$ and $S$ is called a $p$-graded second submodule. The graded second spectrum of $M$, denoted by $\operatorname{Spec}_{G}^{s}(M)$, is the set of all graded second submodules of $M$. For $p \in \operatorname{Spec}_{G}(R), p$ $\operatorname{Spec}_{G}^{s}(M)$ denotes the collection of all $p$-graded second submodules, i.e. $p$ $\operatorname{Spec}_{G}^{s}(M)=\left\{S \in \operatorname{Spec}_{G}^{s}(M) \mid \operatorname{Ann}_{R}(S)=p\right\}$. Graded second submodules of graded modules have been studied by various authors (see, for example, [5, 8, 13]).

A non-zero graded submodule $N$ of a graded $R$-module $M$ is said to be graded large if for every non-zero graded submodule $L$ of $M$, we have $N \cap L \neq 0$. A graded $R$-module $M$ is said to be graded simple if $\{0\}$ and $M$ are its only graded submodules. A non-zero graded submodule $N$ of a graded $R$-module $M$ is called graded minimal if it contains no other non-zero graded submodules of $M$. Equivalently, $N$ is a non-zero graded submodule of $M$ which is a graded simple module. The set of all graded minimal submodules of a $G$-graded $R$-module $M$ will be denoted by $\operatorname{Min}_{G}(M)$. The graded socle of a $G$-graded $R$-module $M$, denoted by $\operatorname{Soc}_{G}^{R}(M)$, is defined to be the sum 
of all graded minimal submodules of $M$. If there are no graded minimal submodules of $M$, we put $\operatorname{Soc}_{G}^{R}(M)=\{0\}$, see 9 . A graded $R$-module $M$ is said to be graded cocyclic if $\operatorname{Soc}_{G}^{R}(M)$ is a graded large and a graded simple submodule of $M$, see 6 .

The Zariski-topology on some graded spectrums of graded rings and graded modules has been studied by several authors (see, for example, [3, 4, 10, 11, 14 ).

In this paper, we introduce the notion of the $G$-cotop module which is analogous to the $G$-top module in [10] and the primary $G$-top module in [14. For this, we define the variety of any graded submodule $N$ of a $G$-graded $R$-module $M$ by $V_{G}^{s *}(N)=\left\{S \in \operatorname{Spec}_{G}^{s}(M) \mid S \subseteq N\right\}$ and we set $\Omega^{s *}(M)=\left\{V_{G}^{s *}(N) \mid N \leq_{G} M\right\}$. Then $M$ is called a $G$-cotop module if $\Omega^{s *}(M)$ is closed under finite union. In this case, $\Omega^{s *}(M)$ generates a topology. We call it the quasi-Zariski topology on $\operatorname{Spec}_{G}^{s}(M)$. After this, we define another variety for any $N \leq_{G} M$ by $V_{G}^{s}(N)=\left\{S \in \operatorname{Spec}_{G}^{s}(M)\right.$ $\left.A n n_{R}(N) \subseteq A n n_{R}(S)\right\}$. Then the collection $\Omega^{s}(M)=\left\{V_{G}^{s}(N) \mid N \leq_{G} M\right\}$ satisfies the axioms for closed sets of a topology on $\operatorname{Spec}_{G}^{s}(M)$ and we call this topology the Zariski topology on $\operatorname{Spec}_{G}^{s}(M)$. By [13, the map $\phi$ : $\operatorname{Spec}_{G}^{s}(M) \rightarrow \operatorname{Spec}_{G}\left(R / A n n_{R}(M)\right)$ defined by $\phi(S)=A n n_{R}(S) / A n n_{R}(M)$ is called the natural map of $\operatorname{Spec}_{G}^{s}(M)$. We show that $\phi$ is continuous and we investigate some conditions under which $\phi$ is injective, open and closed. In addition, using this map, we give some relations between the properties of $\operatorname{Spec}_{G}^{s}(M)$ and $\operatorname{Spec}_{G}\left(R / A n n_{R}(M)\right)$ such as the connectedness. Furthermore, we find a base for the Zariski-topology on $\operatorname{Spec}_{G}^{s}(M)$ and we investigate $\operatorname{Spec}_{G}^{s}(M)$ with the Zariski-topology from the viewpoint of being quasi-compact, irreducible, $T_{0}$-space and $T_{1}$-space.

Throughout this paper, $G$ is a multiplicative group and every ring is commutative with identity. Let $M$ be a $G$-graded $R$-module. For any graded ideal $I$ of $R$ containing $A n n_{R}(M), \bar{I}$ and $\bar{R}$ will denote $I / A n n_{R}(M)$ and $R / A n n_{R}(M)$, respectively. In addition, we denote $r+A n n_{R}(M)$ by $\bar{r}$ for any $r \in R$.

\section{Topologies on $\operatorname{Spec}_{G}^{s}(M)$}

In this section, we first introduce the notion of the $G$-cotop modules and discover some properties of such modules. Then, we define a variety of any graded submodule of a graded module and we use it to define the Zariskitopology on $\operatorname{Spec}_{G}^{s}(M)$.

Proposition 2.1. Let $M$ be a $G$-graded $R$-module. For any $G$-graded submodule $N$ of $M$, we define the variety of $N$ by $V_{G}^{s *}(N)=\left\{S \in \operatorname{Spec}_{G}^{s}(M) \mid\right.$ $S \subseteq N\}$. Then the following hold:

(1) $V_{G}^{s *}(M)=\operatorname{Spec}_{G}^{s}(M)$ and $V_{G}^{s *}(0)=\emptyset$.

(2) $\bigcap_{i \in I} V_{G}^{s *}\left(N_{i}\right)=V_{G}^{s *}\left(\bigcap_{i \in I} N_{i}\right)$ for any index set I and any family of graded submodules $\left\{N_{i}\right\}_{i \in I}$. 
(3) $V_{G}^{s *}(N) \cup V_{G}^{s *}\left(N^{\prime}\right) \subseteq V_{G}^{s *}\left(N+N^{\prime}\right)$ for any $N, N^{\prime} \leq_{G} M$.

Proof. The proof is straightforward.

By Proposition 2.1, the collection $\Omega^{s *}(M)=\left\{V_{G}^{s *}(N) \mid N \leq_{G} M\right\}$ satisfies the axioms for closed sets of a topology on $\operatorname{Spec}_{G}^{s}(M)$ if and only if $\Omega^{s *}(M)$ is closed under finite union. In this case, we call $M$ a $G$-cotop module and the generated topology, $\tau_{G}^{s *}$, the quasi-Zariski topology on $\operatorname{Spec}_{G}^{s}(M)$.

Theorem 2.2. Every comultiplication $G$-graded $R$-module $M$ is a $G$-cotop module.

Proof. Let $N, N^{\prime} \leq_{G} M$ and $S \in V_{G}^{s *}\left(N+N^{\prime}\right)$. It is enough to prove that $S \in V_{G}^{s *}(N) \cup V_{G}^{s *}\left(N^{\prime}\right)$. Assume that $S \nsubseteq N$ and let $s \in S$. Since $S \nsubseteq N$ and $M$ is a comultiplication graded module, then $\exists a \in S$ such that $a \notin N=A n n_{M}\left(A n n_{R}(N)\right)$ and thus $\exists r \in h(R) \cap A n n_{R}(N)$ such that $r a \neq 0$. Since $S \in \operatorname{Spec}_{G}^{s}(M)$ and $r S \neq 0$, then $r S=S$ and hence $s=r m$ for some $m \in S$. But $S \subseteq N+N^{\prime}$. This follows that $m=n+n^{\prime}$ for some $n \in N$ and $n \in N^{\prime}$. So $s=r\left(n+n^{\prime}\right)=r n^{\prime}$ as $r \in A n n_{R}(N)$ and so $s \in N^{\prime}$. This implies that $S \subseteq N$ or $S \subseteq N^{\prime}$. Therefore $S \in V_{G}^{s *}(N) \cup V_{G}^{s *}\left(N^{\prime}\right)$.

Recall that an $R$-module $M$ is called secondless if it has no second submodules. On the other hand, a $G$-graded $R$-module $M$ is said to be graded secondless if it has no graded second submodules ( i.e $\operatorname{Spec}_{G}^{s}(M)=\emptyset$ ). For a graded submodule $N$ of a $G$-graded $R$-module $M$, the graded second socle (or graded second radical) of $N$ is defined as the sum of all graded second submodules of $M$ contained in $N$ and it is denoted by $\operatorname{soc}_{G}(N)$ (or $\sec _{G}(N)$ ). If $N$ does not contain any graded second submodule, we put $\operatorname{soc}_{G}(N)=\{0\}$. Moreover, $N$ is called a graded socle submodule if $N \neq 0$ and $\operatorname{soc}_{G}(N)=N$ (see 13$]$ ).

Remark 2.3. If a $G$-graded $R$-module $M$ is graded secondless, then $M$ is trivially a $G$-cotop module.

Lemma 2.4. Let $M$ be a $G$-graded $R$-module. Let $N$ be a G-graded $R$ submodule of $M$ and $p$ be a $G$-graded ideal of $R$. Then the following hold:

(1) $N$ is a graded second $R$-submodule of $M$ if and only if $N$ is a graded second $\bar{R}$-submodule of $M$.

(2) If $S$ is a G-graded second $R / p$-submodule of $A n n_{M}(p)$, then $S$ is a $G$-graded second $\bar{R}$-submodule of $\operatorname{Ann}_{M}(p)$.

(3) $V_{G}^{s *}(N)=V_{G}^{s *}\left(\operatorname{soc}_{G}(N)\right)$.

(4) If $p \in \operatorname{Spec}_{G}(R)$ and $N^{\prime} \in p-\operatorname{Spec}_{G}^{s}(M)$, then $N^{\prime} \subseteq N$ or $(N+$ $\left.N^{\prime}\right) / N \in p-\operatorname{Spec}_{G}^{s}(M / N)$.

Proof. (1) follows from [8, Proposition 2.4] and (2) is obvious.

(3) Since $\operatorname{soc}_{G}(N) \subseteq N$, then $V_{G}^{s *}\left(\operatorname{soc}_{G}(N)\right) \subseteq V_{G}^{s *}(N)$. Let $S \in V_{G}^{s *}(N)$. Then $S \in \operatorname{Spec}_{G}^{s}(M)$ and $S \subseteq N$. So $S=\operatorname{soc}_{G}(S) \subseteq \operatorname{soc}_{G}(N)$ and thus $S \in V_{G}^{s *}\left(\operatorname{soc}_{G}(N)\right)$.

(4) Assume that $N^{\prime} \nsubseteq N$. Then $\left(N+N^{\prime}\right) / N \in \operatorname{Spec}_{G}^{s}(M / N)$. It is clear that 
$p \subseteq A n n_{R}\left(\left(N+N^{\prime}\right) / N\right)$. Let $r \in A n n_{R}\left(\left(N+N^{\prime}\right) / N\right) \cap h(R)$. By hypothesis, $r N^{\prime}=0$ or $r N^{\prime}=N^{\prime}$. If $r N^{\prime}=N^{\prime}$, then $N^{\prime} \subseteq N$ which is a contradiction. Thus $r \in \operatorname{Ann}_{R}\left(N^{\prime}\right)=p$. Therefore $\operatorname{Ann}_{R}\left(\left(N+N^{\prime}\right) / N\right)=p$.

Proposition 2.5. Let $M$ be a $G$-graded $R$-module. Then the following hold:

(1) If $M_{g}$ is a secondless $R_{e}$-module for every $g \in G$, then $M$ is a graded secondless $R$-module. The converse is true if $R$ is trivially graded by $G$.

(2) If $N \leq_{G} M$ such that both $N$ and $M / N$ are graded secondless $R$ modules, then $M$ is a graded secondless $R$-module.

Proof. (1) Suppose that $M$ is not a graded secondless $R$-module. Then $\exists S \in \operatorname{Spec}_{G}^{s}(M)$. Since $S \neq 0$, then $\exists g \in G$ such that $S_{g} \neq 0$. By [8, Theorem 2.3], $S_{g}$ is a second $R_{e}$-submodule of $M_{g}$, a contradiction. Conversely, assume by way of contradiction that $\exists t \in G$ such that $M_{t}$ is not a secondless $R_{e}$-module which implies that there is a second $R_{e}$-submodule $H$ of $M_{g}$. Since $R$ is trivially graded by $G$, then it is easy to see that $H \in \operatorname{Spec}_{G}^{s}(M)$ which is also a contradiction.

(2) Suppose that $M$ is not graded secondless. Then $\exists S \in \operatorname{Spec}_{G}^{s}(M)$. Let $p=A n n_{R}(S)$. So we get $S \in p-\operatorname{Spec}_{G}^{s}(M)$. Since $M / N$ is graded secondless, then $(N+S) / N \notin p$-Spec $G_{G}^{s}(M)$ and hence $S \subseteq N$ by Lemma 2.4(4). Therefore $S \in \operatorname{Spec}_{G}^{s}(N)=\emptyset$, a contradiction.

If we take the ring of integers $R=\mathbb{Z}$ as a $\mathbb{Z}_{2}$-graded $\mathbb{Z}$-module by $R_{0}=R$ and $R_{1}=\{0\}$, then $R_{0}$ and $R_{1}$ are secondless $R_{0}$-module which follows that $\mathbb{Z}$ is a $\mathbb{Z}_{2}$-graded secondless $\mathbb{Z}$-module by Proposition 2.5 .

A graded second submodule $S$ of a graded $R$-module $M$ is said to be graded extraordinary if whenever $N_{1}$ and $N_{2}$ are graded socle submodules of $M$ with $S \subseteq N_{1}+N_{2}$, then $S \subseteq N_{1}$ or $S \subseteq N_{2}$.

Theorem 2.6. Let $M$ be a $G$-graded $R$-module. Then the following statements are equivalent:

(1) $M$ is a $G$-cotop module.

(2) Every graded second submodule of $M$ is graded extraordinary.

(3) $V_{G}^{s *}(N) \cup V_{G}^{s *}\left(N^{\prime}\right)=V_{G}^{s *}\left(N+N^{\prime}\right)$ for any graded socle submodules $N, N^{\prime}$ of $M$.

Proof. If $M$ is graded secondless, then the result is trivially true. Suppose that $M$ is not graded secondless.

$(1) \Rightarrow(2)$ : Let $S \in \operatorname{Spec}_{G}^{s}(M)$ and let $N$ and $L$ be graded socle submodules of $M$ such that $S \subseteq N+L$. By hypothesis, $V_{G}^{s *}(N) \cup V_{G}^{s *}(L)=V_{G}^{s *}(H)$ for some $H \leq_{G} M$. Now there is an index set $I$ such that $N=\sum_{i \in I} S_{i}$, where $S_{i} \in \operatorname{Spec}_{G}^{s}(M)$ and $S_{i} \subseteq N$ for every $i \in I$. For each $i \in I, S_{i} \in$ $V_{G}^{s *}(N) \subseteq V_{G}^{s *}(H)$ and hence $S_{i} \subseteq H$ which implies that $N \subseteq H$. Similarly $L \subseteq H$. Thus $N+L \subseteq H$. So $V_{G}^{s *}(N) \cup V_{G}^{s *}(L) \subseteq V_{G}^{s *}(N+L) \subseteq V_{G}^{s *}(H)=$ $V_{G}^{s *}(N) \cup V_{G}^{s *}(L)$ and so $V_{G}^{s *}(N+L)=V_{G}^{s *}(N) \cup V_{G}^{s *}(L)$. But $S \in V_{G}^{s *}(N+L)$. Therefore $S \subseteq N$ or $S \subseteq L$, as desired. 
$(2) \Rightarrow(3)$ : It is clear.

$(3) \Rightarrow(1)$ : Let $W, W^{\prime} \leq_{G} M$. By Lemma 2.4(3), we have $V_{G}^{s *}(W) \cup V_{G}^{s *}\left(W^{\prime}\right)=$ $V_{G}^{s *}\left(\operatorname{soc}_{G}(W)\right) \cup V_{G}^{s *}\left(\operatorname{soc}_{G}\left(W^{\prime}\right)\right)$. If $\operatorname{soc}_{G}(W)=0$ or $\operatorname{soc}_{G}\left(W^{\prime}\right)=0$, then the result is trivially true. If not, then $\operatorname{soc}_{G}(W)$ and $\operatorname{soc}_{G}\left(W^{\prime}\right)$ are graded socle submodules of $M$. By hypothesis, $V_{G}^{s *}(W) \cup V_{G}^{s *}\left(W^{\prime}\right)=V_{G}^{s *}\left(\operatorname{soc}_{G}(W)+\right.$ $\left.\operatorname{soc}_{G}\left(W^{\prime}\right)\right)$. Therefore $M$ is a $G$-cotop module.

Corollary 2.7. $\quad$ (1) Every graded submodule $N$ of a $G$-cotop module $M$ is a $G$-cotop module.

(2) $M$ is a $G$-cotop $R$-module if and only if $M$ is a $G$-cotop $\bar{R}$-module.

(3) Let $M$ be a G-graded $R$-module and $p \triangleleft_{G} R$. If $M$ is a $G$-cotop $R$-module, then $A n n_{M}(p)$ is a $G$-cotop $R / p$-module.

(4) Let $H$ be a graded subring of a graded ring $R$ and let $M$ be a $G$-graded $R$-module such that it is a $G$-cotop as graded $H$-module. Then $M$ is a $G$-cotop $R$-module.

(5) Let $f: M \rightarrow M^{\prime}$ be a G-graded module isomorphism of G-graded $R$ modules. If $M$ is a $G$-cotop module, then $M^{\prime}$ is a $G$-cotop module.

Proof. (1) Let $S \in \operatorname{Spec}_{G}^{s}(N)$ and $L_{1}, L_{2}$ be graded socle submodules of $N$ such that $S \subseteq L_{1}+L_{2}$. Clearly, $\operatorname{Spec}_{G}^{s}(N) \subseteq \operatorname{Spec}_{G}^{s}(M)$. So $S \in \operatorname{Spec}_{G}^{s}(M)$. Now it is easy to see that $L_{1}, L_{2}$ are graded socle submodules of $M$. Since $M$ is a $G$-cotop module, then $S \subseteq L_{1}$ or $S \subseteq L_{2}$ by Theorem 2.6. Therefore $N$ is a $G$-cotop module by Theorem 2.6 again.

(2) If $M$ is a graded secondless $R$-module, then $M$ is a graded secondless $\bar{R}$-module by Lemma $2.4(1)$. Thus the result is clearly true by Remark 2.3 . Suppose that $M$ is not a graded secondless $R$-module. Then by Lemma 2.4(1) and Theorem 2.6, we have $M$ is a $G$-cotop module $\Leftrightarrow$ Every graded second $R$-submodule of $M$ is graded extraordinary $\Leftrightarrow$ Every graded second $\bar{R}$-submodule of $M$ is graded extraordinary $\Leftrightarrow M$ is a $G$-cotop $\bar{R}$-module.

(3) Suppose that $A n n_{M}(p)$ is not a graded secondless $R / p$-module. Let $S$ be a graded second $R / p$-submodule of $\operatorname{Ann}_{M}(p)$ and $N, K$ be graded socle $R / p$-submodules of $A n n_{M}(p)$ with $S \subseteq N+K$. By Lemma 2.4(2), $S$ is a graded second $\bar{R}$-submodule of $A n n_{M}(p)$. Also, it is easy to see that $N, K$ are graded socle $\bar{R}$-submodules of $A n n_{M}(p)$. But $M$ is a $G$-cotop $R$-module. So $A n n_{M}(p)$ is a $G$-cotop $R$-module by (1) and hence it is a $G$-cotop $\bar{R}$-module by (2). This implies that $S \subseteq N$ or $S \subseteq K$ by Theorem 2.6. Therefore $A n n_{M}(p)$ is a $G$-cotop $R / p$-modue by Theorem 2.6 again.

(4) Suppose that $M$ is not a graded secondless $R$-module. Let $S$ be a graded second $R$-submodule of $M$ and $W_{1}, W_{2}$ be graded socle $R$-submodules of $M$ such that $S \subseteq W_{1}+W_{2}$. It is straightforward to see that $S$ is a graded second $H$-submodule of $M$ and $W_{1}, W_{2}$ are graded socle $H$-submodules of $M$. But $M$ is a $G$-cotop $H$-module. By Theorem 2.6, $S \subseteq W_{1}$ or $S \subseteq W_{2}$. Therefore $M$ is a $G$-cotop $R$-module.

(5) Suppose that $M^{\prime}$ is not graded secondless. Let $S^{\prime} \in \operatorname{Spec}_{G}^{s}\left(M^{\prime}\right)$ and $N_{1}^{\prime}, N_{2}^{\prime}$ be graded socle submodules of $M^{\prime}$ such that $S^{\prime} \subseteq N_{1}^{\prime}+N_{2}^{\prime}$. Since $f$ is a graded module isomorphism, then $f^{-1}\left(S^{\prime}\right) \in \operatorname{Spec}_{G}^{s}(M)$ and $f^{-1}\left(N_{1}^{\prime}\right), f^{-1}\left(N_{2}^{\prime}\right)$ 
are graded socle submodules of $M$. Now $f^{-1}\left(S^{\prime}\right) \subseteq f^{-1}\left(N_{1}^{\prime}+N_{2}^{\prime}\right)=$ $f^{-1}\left(N_{1}^{\prime}\right)+f^{-1}\left(N_{2}^{\prime}\right)$. But $M$ is a $G$-cotop module. Hence $f^{-1}\left(S^{\prime}\right) \subseteq f^{-1}\left(N_{1}^{\prime}\right)$ or $f^{-1}\left(S^{\prime}\right) \subseteq f^{-1}\left(N_{2}^{\prime}\right)$. By the surjectivity of $f$, we obtain $S^{\prime} \subseteq N_{1}^{\prime}$ or $S^{\prime} \subseteq N_{2}^{\prime}$. Therefore $M^{\prime}$ is a $G$-cotop module.

A graded ring $F$ is called a graded field if every non-zero homogeneous element of $F$ is unit. As usual, the set of all units of a graded ring $R$ will be denoted by $U(R)$. A graded $R$-module $M$ is said to be graded cyclic if there exists $m \in h(M)$ such that $M=R m$, see 9 .

Lemma 2.8. Let $M$ be a $G$-graded $R$-module. Then the following hold:

(1) If $R$ is a graded field, then every non-zero graded submodule of $M$ is graded second.

(2) If $A \subseteq h(M)$ is a base for $M$ such that $|A|>1$, then there exist non-zero graded submodules $N_{1}$ and $N_{2}$ of $M$ such that $N_{1} \nsubseteq N_{2}$ and $N_{2} \nsubseteq N_{1}$.

(3) If $R$ is a graded field and $M$ is a non-zero graded cyclic $R$-module, then $M$ is a graded simple $R$-module.

Proof. (1) Let $N$ be a non-zero graded submodule of $M$. Let $r \in h(R)$. If $r=0$, then $r N=0$. If $r \neq 0$, then $r \in U(R)$. So for any $n \in N$, we have $n=r\left(r^{-1} n\right) \in r N$ and thus $r N=0$ or $r N=N$. Therefore $N \in \operatorname{Spec}_{G}^{s}(M)$. (2) Assume by way of contradiction that for any non-zero graded submodules $N_{1}$ and $N_{2}$ of $M$, we have $N_{1} \subseteq N_{2}$ or $N_{2} \subseteq N_{1}$. By hypothesis, there exist $m_{1}, m_{2} \in A$ such that $m_{1} \neq m_{2}$. It is clear that $m_{1}, m_{2} \neq 0$ as $A$ is a base for $M$. Since $A \subseteq h(M)$, then $R m_{1}$ and $R m_{2}$ are non-zero graded submodules of $M$. Hence $R m_{1} \subseteq R m_{2}$ or $R m_{2} \subseteq R m_{1}$. If $R m_{1} \subseteq R m_{2}$, then $m_{1}=r m_{2}$ for some $r \in R$ and thus $m_{1}+(-r) m_{2}=0$. Since $A$ is a linearly independent set, then $r=0$ and thus $m_{1}=0$, a contradiction. Similarly, if $R m_{2} \subseteq R m_{1}$, we also have a contradiction.

(3) By hypothesis, $M=R m$ for some $m \in h(M)$. Let $x \in M$ and $m^{\prime} \in$ $h(M)-\{0\}$. Then $x=r m$ and $m^{\prime}=c m$ for some $r, c \in R$. Now $m^{\prime} \in M_{g}$ and $m \in M_{h}$ for some $g, h \in G$. So $m^{\prime}=c_{g h^{-1}} m$. Let $a=c_{g h^{-1}}$. Since $m^{\prime} \neq 0$, then $a \neq 0$ and thus $a \in U(R)$. This implies that $m=a^{-1} m^{\prime}$ and hence $x=r\left(a^{-1} m^{\prime}\right) \in R m^{\prime}$. So we obtain $M=R m^{\prime}$. This means that $M$ is a non-zero graded cyclic module generated by any non-zero homogeneous element. Therefore $M$ is a graded simple $R$-module.

Corollary 2.9. Let $M$ be a G-graded module over a graded field $F$. If $A \subseteq h(M)$ is a base for $M$, then $M$ is a G-cotop module if and only if $|A| \leq 1$.

Proof. $\Rightarrow$ : Suppose that $|A|>1$. By Lemma 2.8(2), there exist non-zero graded submodules $N_{1}$ and $N_{2}$ of $M$ such that $N_{1} \nsubseteq N_{2}$ and $N_{2} \nsubseteq N_{1}$. Also $N_{1}, N_{2}, N_{1}+N_{2} \in \operatorname{Spec}_{G}^{s}(M)$ by Lemma 2.8(1). Thus $N_{1}=\operatorname{soc}_{G}\left(N_{1}\right)$ and $N_{2}=\operatorname{soc}_{G}\left(N_{2}\right)$. Since $M$ is a $G$-cotop module and $N_{1}+N_{2} \subseteq N_{1}+N_{2}$, then $N_{1} \subseteq N_{2}$ or $N_{2} \subseteq N_{1}$ by Theorem 2.6, a contradiction. 
$\Leftarrow$ : If $M=0$, then $M$ is a graded secondless module and hence it a $G$-cotop module by Remark 2.3 . If $M \neq 0$, then $|A|=1$ and thus $M$ is a graded simple $F$-module by Lemma $2.8(3)$. Therefore $M$ is a $G$-cotop module.

The following is an example on a non $G$-cotop module.

Example 2.10. Let $F$ be any field. Take $G=Z_{2}$ and $M=F \times F$. Then $F$ is a $G$-graded field by $F_{0}=F$ and $F_{1}=\{0\}$. Also $M$ is a $G$-graded $F$-module by $M_{0}=F \times\{0\}$ and $M_{1}=\{0\} \times F$. Moreover $M_{0}, M_{1} \leq_{G} M$. Since $F$ is a $G$-graded field, then $M \in \operatorname{Spec}_{G}^{s}(M)$ and $M_{0}, M_{1}$ are graded socle submodules of $M$ by Lemma 2.8(1). Now $M \subseteq M_{0}+M_{1}$. But $M \nsubseteq M_{0}$ and $M \nsubseteq M_{1}$. Therefore $M$ is not a $G$-cotop module by Theorem 2.6.

Let $M$ be a $G$-cotop module and $Y \subseteq \operatorname{Spec}_{G}^{s}(M)$. The closure of $Y$ in $\operatorname{Spec}_{G}^{s}(M)$ for the quasi-Zariski topology will be expressed by $C l(Y)$.

Lemma 2.11. Let $M$ be a $G$-cotop module and $Y \subseteq \operatorname{Spec}_{G}^{s}(M)$. Let $T(Y)=$ $\sum_{S \in Y} S$. Then $C l(Y)=V_{G}^{s *}(T(Y))$. In particular, if $M \in Y$, then $Y$ is dense in $\operatorname{Spec}_{G}^{s}(M)$, that is, $C l(Y)=\operatorname{Spec}_{G}^{s}(M)$.

Proof. It is clear that $Y \subseteq V_{G}^{s *}(T(Y))$. Let $V_{G}^{s *}(N)$ be any closed set containing $Y$, where $N \leq_{G} M$. It is enough to show that $V_{G}^{s *}(T(Y)) \subseteq V_{G}^{s *}(N)$. Let $S \in V_{G}^{s *}(T(Y))$. Then $S \subseteq T(Y)$. Note that for any $S^{\prime} \in Y$, we have $S^{\prime} \subseteq N$ and hence $T(Y) \subseteq N$. This implies that $S \subseteq N$ and thus $S \in V_{G}^{s *}(N)$. Therefore $C l(Y)=V_{G}^{s *}(T(Y))$. Now if $M \in Y$, then $C l(Y)=V_{G}^{s *}(T(Y))=V_{G}^{s *}(M)=\operatorname{Spec}_{G}^{s}(M)$.

Corollary 2.12. Let $M$ be a $G$-cotop module. Then $\operatorname{Spec}_{G}^{s}(M)$ is a $T_{0}$ space.

Proof. Let $S_{1}, S_{2} \in \operatorname{Spec}_{G}^{s}(M)$ with $S_{1} \neq S_{2}$. If $C l\left(\left\{S_{1}\right\}\right)=C l\left(\left\{S_{2}\right\}\right)$, then $V_{G}^{s *}\left(S_{1}\right)=V_{G}^{s *}\left(S_{2}\right)$ by Lemma 2.11. This implies that $S_{1}=S_{2}$, a contradiction. Thus $C l\left(\left\{S_{1}\right\}\right) \neq C l\left(\left\{S_{2}\right\}\right)$. This means that the closures of any two distinct points of $\operatorname{Spec}_{G}^{s}(M)$ are distinct. Therefore $\operatorname{Spec}_{G}^{s}(M)$ is a $T_{0}$-space.

Lemma 2.13. Let $M$ be a $G$-graded $R$-module. If $N \leq_{G} M$ and $I \triangleleft_{G} R$, then $V_{G}^{s *}(N) \cup V_{G}^{s *}\left(\operatorname{Ann}_{M}(I)\right)=V_{G}^{s *}\left(\left(N:_{M} I\right)\right)$.

Proof. It is clear that $V_{G}^{s *}(N) \cup V_{G}^{s *}\left(A n n_{M}(I)\right) \subseteq V_{G}^{s *}\left(\left(N:_{M} I\right)\right)$. Let $S \in V_{G}^{s *}\left(\left(N:_{M} I\right)\right)$. Suppose that $S \nsubseteq N$ and it is enough to show that $S \subseteq \operatorname{Ann}_{M}(I)$. Let $s \in S$ and $i \in I$. Now $S \subseteq\left(N:_{M} I\right)$. So $i S \subseteq N$. Since $S \in \operatorname{Spec}_{G}^{s}(M)$, then $i S=\{0\}$ and hence $i s=0$, as desired.

Corollary 2.14. Let $M$ be a $G$-graded $R$-module. If $I, J \triangleleft_{G} R$, then $V_{G}^{s *}\left(A n n_{M}(I)\right) \cup$ $V_{G}^{s *}\left(A n n_{M}(J)\right)=V_{G}^{s *}\left(A n n_{M}(I J)\right)$.

Proof. Clearly, $\left(A n n_{M}(I):_{M} J\right)=A n n_{M}(I J)$. By Lemma 2.13, $V_{G}^{s *}\left(A n n_{M}(I)\right) \cup$ $V_{G}^{s *}\left(\operatorname{Ann}_{M}(J)\right)=V_{G}^{s *}\left(\left(A n n_{M}(I):_{M} J\right)\right)=V_{G}^{s *}\left(A n n_{M}(I J)\right)$. 
Let $M$ be a $G$-graded $R$-module. By Corollary 2.14, the family $\Omega^{s \prime}=$ $\left\{V_{G}^{s *}\left(A n n_{M}(I)\right) \mid I \triangleleft_{G} R\right\}$ always induce a topology on $\operatorname{Spec}_{G}^{s}(M)$ and it is denoted by $\tau_{G}^{S \prime}$. Now, the proof of the following proposition is obvious.

Proposition 2.15. Let $M$ be a G-graded $R$-module. If $M$ is a G-cotop module, then the quasi-Zariski topology $\tau_{G}^{s *}$ on $S p e c_{G}^{s}(M)$ is finer than $\tau_{G}^{s \prime}$.

Now, we define another variety for a graded submodule $N$ of a $G$-graded $R$-module $M$. We set $V_{G}^{s}(N)=\left\{S \in \operatorname{Spec}_{G}^{s}(M) \mid A n n_{R}(N) \subseteq A n n_{R}(S)\right\}$. We state some properties of this variety in the following theorem to construct the Zariski-topology on $\operatorname{Spec}_{G}^{s}(M)$.

Theorem 2.16. Let $M$ be a $G$-graded $R$-module. Then the following hold:

(1) $V_{G}^{s}(M)=\operatorname{Spec}_{G}^{s}(M)$ and $V_{G}^{s}(0)=\emptyset$.

(2) $\bigcap_{i \in I} V_{G}^{s}\left(N_{i}\right)=V_{G}^{s}\left(\bigcap_{i \in I} A n n_{M}\left(A n n_{R}\left(N_{i}\right)\right)\right)$, for any family $\left\{N_{i}\right\}_{i \in I}$ of graded submodules.

(3) $V_{G}^{s}(N) \cup V_{G}^{s}\left(N^{\prime}\right)=V_{G}^{s}\left(N+N^{\prime}\right)$ for any graded submodules $N, N^{\prime}$ of $M$.

Proof. (1) It is clear.

(2) Let $S \in \bigcap_{i \in I} V_{G}^{s}\left(N_{i}\right)$. For all $i \in I$, we have $A n n_{R}\left(N_{i}\right) \subseteq A n n_{R}(S)$ and thus $\operatorname{Ann}_{M}\left(\operatorname{Ann}_{R}(S)\right) \subseteq A n n_{M}\left(\operatorname{Ann}_{R}\left(N_{i}\right)\right)$. Hence $\operatorname{Ann}_{M}\left(\operatorname{Ann} n_{R}(S)\right) \subseteq$ $\bigcap_{i \in I} A n n_{M}\left(A n n_{R}\left(N_{i}\right)\right)$. It follows that $A n n_{R}\left(\bigcap_{i \in I} A n n_{M}\left(A n n_{R}\left(N_{i}\right)\right)\right) \subseteq A n n_{R}\left(A n n_{M}\left(A n n_{R}(S)\right)\right)=$ $A n n_{R}(S)$. So $S \in V_{G}^{s}\left(\bigcap_{i \in I} A n n_{M}\left(A n n_{R}\left(N_{i}\right)\right)\right)$ and so $\bigcap_{i \in I} V_{G}^{s}\left(N_{i}\right) \subseteq V_{G}^{s}\left(\bigcap_{i \in I} A n n_{M}\left(A n n_{R}\left(N_{i}\right)\right)\right)$.

Conversely, let $S \in V_{G}^{s}\left(\bigcap_{i \in I} A n n_{M}\left(A n n_{R}\left(N_{i}\right)\right)\right)$. Then $A n n_{R}\left(\bigcap_{i \in I} A n n_{M}\left(A n n_{R}\left(N_{i}\right)\right)\right) \subseteq$ $A n n_{R}(S)$. For all $i \in I$, we have $\bigcap_{i \in I} A n n_{M}\left(A n n_{R}\left(N_{i}\right)\right) \subseteq A n n_{M}\left(A n n_{R}\left(N_{i}\right)\right)$ and hence $A n n_{R}\left(N_{i}\right)=A n n_{R}\left(A n n_{M}\left(A n n_{R}\left(N_{i}\right)\right)\right) \subseteq A n n_{R}\left(\bigcap_{i \in I} A n n_{M}\left(A n n_{R}\left(N_{i}\right)\right)\right) \subseteq$ $A n n_{R}(S)$. Therefore $S \in \bigcap_{i \in I} V_{G}^{s}\left(N_{i}\right)$.

(3) Since $N, N^{\prime} \subseteq N+N^{\prime}$, then $V_{G}^{s}(N) \subseteq V_{G}^{s}\left(N+N^{\prime}\right)$ and $V_{G}^{s}\left(N^{\prime}\right) \subseteq$ $V_{G}^{s}\left(N+N^{\prime}\right)$ and thus $V_{G}^{s}(N) \cup V_{G}^{s}\left(N^{\prime}\right) \subseteq V_{G}^{s}\left(N+N^{\prime}\right)$. For the reverse inclusion, let $S \in V_{G}^{s}\left(N+N^{\prime}\right)$. Then $\operatorname{Ann}_{R}\left(N+N^{\prime}\right) \subseteq \operatorname{Ann}_{R}(S)$. So we get $A n n_{R}(N) A n n_{R}\left(N^{\prime}\right) \subseteq A n n_{R}(S) \in \operatorname{Spec}_{G}(R)$. By 11, Proposition 1.2], we have $A n n_{R}(N) \subseteq A n n_{R}(S)$ or $A n n_{R}\left(N^{\prime}\right) \subseteq A n n_{R}(S)$. Therefore $S \in V_{G}^{s}(N) \cup V_{G}^{s}\left(N^{\prime}\right)$.

In view of Theorem 2.16, the collection $\Omega^{s}(M)=\left\{V_{G}^{s}(N) \mid N \leq_{G} M\right\}$ satisfies the axioms for closed sets of a topology on $\operatorname{Spec}_{G}^{s}(M)$. This topology is called the Zariski-topology on $\operatorname{Spec}_{G}^{S}(M)$ and it is denoted by $\tau_{G}^{s}$.

In the following lemma, we state some relations between the varieties $V_{G}^{s *}(N)$ and $V_{G}^{s}(N)$ for any graded submodule $N$ of a $G$-graded $R$-module $M$.

Lemma 2.17. Suppose that $N$ and $N^{\prime}$ are graded submoduels of a $G$-graded $R$-module $M$ and that $I$ is a $G$-graded ideal of $R$. Then the following hold: 
(1) If $\operatorname{Ann}_{R}(N)=A n n_{R}\left(N^{\prime}\right)$, then $V_{G}^{s}(N)=V_{G}^{s}\left(N^{\prime}\right)$. The converse is also true if $N, N^{\prime} \in \operatorname{Spec}_{G}^{s}(M)$.

(2) $V_{G}^{s}(N)=\bigcup_{p \in V_{G}^{R}\left(\operatorname{Ann}_{R}(N)\right)} p-\operatorname{Spec}_{G}^{s}(M)$

(3) $V_{G}^{s}(N)=V_{G}^{s}\left(A n n_{M}\left(A n n_{R}(N)\right)\right)=V_{G}^{s *}\left(\operatorname{Ann}_{M}\left(A n n_{R}(N)\right)\right)$. In particular, $V_{G}^{s}\left(\operatorname{Ann}_{M}(I)\right)=V_{G}^{s *}\left(\operatorname{Ann}_{M}(I)\right)$.

Proof. The proof is straightforward.

Theorem 2.18. For any $G$-graded $R$-module $M$, the Zariski-topology $\tau_{G}^{s}$ on $\operatorname{Spec}_{G}^{s}(M)$ is identical with $\tau_{G}^{s \prime}$. Moreover, if $M$ is a $G$-cotop module, then the quasi-Zariski topology $\tau_{G}^{s *}$ on $\operatorname{Spec}_{G}^{s}(M)$ is finer than the Zariski-topology $\tau_{G}^{s}$.

Proof. It is clear by Lemma $2.17(3)$.

Proposition 2.19. Let $M$ be a G-graded module over a graded field $F$. If $A \subseteq h(M)$ is a base for $M$ such that $|A| \leq 1$, then $M$ is a $G$-cotop module and $\tau_{G}^{s}=\tau_{G}^{s *}=$ the trivial topology on $\operatorname{Spec}_{G}^{s}(M)$.

Proof. Since $|A| \leq 1$, then $M$ is a $G$-cotop module by Corollary 2.9. It is clear that if $|A|=0$, then $\tau_{G}^{s}=\tau_{G}^{s *}=$ the trivial topology on $\operatorname{Spec}_{G}^{s}(M)$. If $|A|=1$, then $M=F m$ for some $m \in A$. If $m=0$, then $M=0$ and the result is trivially true. Suppose that $m \neq 0$. By Lemma 2.8(3), $M$ is a graded simple $F$-module. Now let $U \in \tau_{G}^{s *}$. Then $U=\operatorname{Spec}_{G}^{s}(M)-V_{G}^{s *}(N)$ for some $N \leq_{G} M$. But $N=0$ or $N=M$. This implies that $\tau_{G}^{s *}=\left\{\emptyset, \operatorname{Spec}_{G}^{s}(M)\right\}$. By Theorem 2.18, $\tau_{G}^{s} \subseteq \tau_{G}^{s *}=\left\{\emptyset, S p e c_{G}^{s}(M)\right\} \subseteq \tau_{G}^{s}$. Therefore $\tau_{G}^{s}=\tau_{G}^{s *}=$ the trivial topology on $\operatorname{Spec}_{G}^{s}(M)$.

\section{Relating $\operatorname{Spec}_{G}^{s}(M)$ And $\operatorname{Spec}_{G}(\bar{R})$}

Let $M$ be a $G$-graded $R$-module. From now on, we assume that $\operatorname{Spec}_{G}^{s}(M)$ is a non-empty set and is equipped the Zariski-topology. We also consider the natural map $\phi$ of $\operatorname{Spec}_{G}^{s}(M)$ as described in the introduction. In this section, we provide some relations between $\operatorname{Spec}_{G}^{s}(M)$ and $\operatorname{Spec}_{G}(\bar{R})$ for any $G$-graded $R$-module $M$.

Proposition 3.1. Let $M$ be a G-graded R-module. Then $\phi^{-1}\left(V_{G}^{\bar{R}}(\bar{I})\right)=$ $V_{G}^{s}\left(\operatorname{Ann}_{M}(I)\right)$, for every graded ideal $I$ of $R$ containing $A n n_{R}(M)$, and therefore the natural map $\phi$ of $\operatorname{Spec}_{G}^{s}(M)$ is continuous.

Proof. For any $S \in \operatorname{Spec}_{G}^{s}(M)$, we have $S \in \phi^{-1}\left(V_{G}^{\bar{R}}(\bar{I})\right) \Leftrightarrow \phi(S)=$ $\overline{A n n_{R}(S)} \in V_{G}^{\bar{R}}(\bar{I}) \Leftrightarrow \bar{I} \subseteq \overline{A n n_{R}(S)} \Leftrightarrow I \subseteq A n n_{R}(S) \Leftrightarrow S \subseteq A n n_{M}(I) \Leftrightarrow$ $A n n_{R}\left(\operatorname{Ann}_{M}(I)\right) \subseteq A n n_{R}(S) \Leftrightarrow S \in V_{G}^{s}\left(A n n_{M}(I)\right)$. Therefore $\phi^{-1}\left(V_{G}^{\bar{R}}(\bar{I})\right)=$ $V_{G}^{s}\left(\operatorname{Ann}_{M}(I)\right)$.

Proposition 3.2. The following are equivalent for any $G$-graded $R$-module $M$ : 
(1) If whenever $S_{1}, S_{2} \in \operatorname{Spec}_{G}^{s}(M)$ with $V_{G}^{s}\left(S_{1}\right)=V_{G}^{s}\left(S_{2}\right)$, then $S_{1}=$ $S_{2}$.

(2) $\left|p-\operatorname{Spec}_{G}^{s}(M)\right| \leq 1$ for every $p \in \operatorname{Spec}_{G}(R)$.

(3) The natural map $\phi$ is injective.

Proof. (1) $\Rightarrow(2)$ : Let $p \in \operatorname{Spec}_{G}(R)$ and $S_{1}, S_{2} \in p-\operatorname{Spec}_{G}^{s}(M)$. Then $S_{1}, S_{2} \in$ $\operatorname{Spec}_{G}^{s}(M)$ and $A n n_{R}\left(S_{1}\right)=A n n_{R}\left(S_{2}\right)=p$. By Lemma 2.17(1), we have $V_{G}^{s}\left(S_{1}\right)=V_{G}^{s}\left(S_{2}\right)$ and hence $S_{1}=S_{2}$ by the assumption (1).

$(2) \Rightarrow(3)$ : Assume that $\phi\left(S_{1}\right)=\phi\left(S_{2}\right)$, where $S_{1}, S_{2} \in \operatorname{Spec}_{G}^{s}(M)$. Then $A n n_{R}\left(S_{1}\right)=A n n_{R}\left(S_{2}\right)$. Let $p=A n n_{R}\left(S_{1}\right) \in \operatorname{Spec}_{G}(R)$. Then we get $S_{1}, S_{2} \in p-\operatorname{Spec}_{G}^{s}(M)$ and by hypothesis, we obtain $S_{1}=S_{2}$.

$(3) \Rightarrow(1)$ : Let $S_{1}, S_{2} \in \operatorname{Spec}_{G}^{s}(M)$ with $V_{G}^{s}\left(S_{1}\right)=V_{G}^{s}\left(S_{2}\right)$. By Lemma $2.17(1)$, we have $A n n_{R}\left(S_{1}\right)=A n n_{R}\left(S_{2}\right)$. So $\phi\left(S_{1}\right)=\phi\left(S_{2}\right)$. Since $\phi$ is injective, then $S_{1}=S_{2}$.

Proposition 3.3. Let $M$ be a $G$-graded $R$-module. If $M$ is a comultiplication graded module, then the natural map $\phi$ of $\operatorname{Spec}_{G}^{s}(M)$ is injective.

Proof. Assume that $\phi\left(S_{1}\right)=\phi\left(S_{2}\right)$, where $S_{1}, S_{2} \in \operatorname{Spec}_{G}^{s}(M)$. Then $A n n_{R}\left(S_{1}\right)=A n n_{R}\left(S_{2}\right)$. Since $M$ is a comultiplication graded module, then $S_{1}=A n n_{M}\left(A n n_{R}\left(S_{1}\right)\right)=A n n_{M}\left(A n n_{R}\left(S_{2}\right)\right)=S_{2}$.

Let $M$ be a $G$-graded $R$-module. Recall that a proper graded submodule $N$ of $M$ is said to be graded completely irreducible if $N=\bigcap_{i \in I} N_{i}$, where $\left\{N_{i}\right\}_{i \in I}$ is a family of graded submodules of $M$, implies that $N=N_{i}$ for some $i \in I$. By [1, Theorem 2.1], every proper graded submodule of $M$ is an intersection of graded completely irreducible submodules of $M$. Therefore, if $M \neq 0$, then the intersection of all graded completely irreducible submodules of $M$ is zero.

Let $M$ and $M^{\prime}$ be $G$-graded $R$-modules. By $M \cong_{G} M^{\prime}$, we mean that there exists a $G$-graded module isomorphism $f: M \rightarrow M^{\prime}$.

Lemma 3.4. Let $M, M^{\prime}$ be $G$-graded $R$-modules and $p \triangleleft_{G} R$. Then the following hold:

(1) If $S$ is a graded second $R / p$-submodule of $A n n_{M}(p)$, then $S$ is a graded second $R$-submodule of $M$.

(2) If $M$ is graded cocyclic and $M \cong_{G} M^{\prime}$, then $M^{\prime}$ is graded cocyclic.

(3) If $M$ is a graded cocyclic $R$-module and $N$ is a non-zero graded submodule of $M$, then $N$ is a graded cocyclic $R$-module.

Proof. (1) By Lemma 2.4(2), $S$ is a graded second $\bar{R}$-submodule of $A n n_{M}(p)$. So $S$ is a graded second $R$-submodule of $A n n_{M}(p)$ by Lemma 2.4(1). Therefore $S$ is a graded second $R$-submodule of $M$.

(2) Since $M \cong_{G} M^{\prime}$, then there exists a $G$-graded module isomorphism $f: M \rightarrow M^{\prime}$. By hypothesis, $\operatorname{Soc}_{G}^{R}(M)$ is a graded large and a graded simple submodule of $M$. Thus $\operatorname{Soc}_{G}^{R}\left(M^{\prime}\right)=f\left(\operatorname{Soc}_{G}^{R}(M)\right)$ is a graded large and a graded simple submodule of $M^{\prime}$. Therefore $M^{\prime}$ is a graded cocyclic 
$R$-module.

(3) Since $N \subseteq M$, then $\operatorname{Soc}_{G}^{R}(N) \subseteq \operatorname{Soc}_{G}^{R}(M)$. By hypothesis, $\operatorname{Soc}_{G}^{R}(M)$ is a graded simple submodule of $M$. So $\operatorname{Soc}_{G}^{R}(N)=0$ or $\operatorname{Soc}_{G}^{R}(N)=$ $\operatorname{Soc}_{G}^{R}(M)$. Since $N \neq 0$ and $\operatorname{Soc}_{G}^{R}(M)$ is a graded large submodule of $M$, then $\operatorname{Soc}_{G}^{R}(M) \cap N \neq 0$. Now, it is easy to see that $\operatorname{Soc}_{G}^{R}(M) \cap N$ is a graded simple submodule of $N$. This follows that $\operatorname{Soc}_{G}^{R}(N) \neq 0$ and thus $\operatorname{Soc}_{G}^{R}(N)=\operatorname{Soc}_{G}^{R}(M)$. But $\operatorname{Soc}_{G}^{R}(N) \subseteq N$. So $\operatorname{Soc}_{G}^{R}(N)$ is a graded large and a graded simple submodule of $N$, as needed.

The following is a result of Proposition 3.2 and Lemma 3.4 .

Corollary 3.5. Let $M$ be a $G$-graded $R$-module. If the natural map $\phi$ is injective, then $A n n_{M}(p)$ is a graded cocyclic module for every $p \in \operatorname{Max}_{G}(R)$ with $\operatorname{Ann}_{M}(p) \neq 0$.

Proof. Let $p \in \operatorname{Max}_{G}(R)$ such that $A n n_{M}(p) \neq 0$. Then $A n n_{M}(p)$ is a graded module over the graded field $R / p$ which follows that $A n n_{M}(p)$ is a graded second $R / p$-submodule of $A n n_{M}(p)$ by Lemma 2.8(1). Thus $A n n_{M}(p)$ is a graded second $R$-submodule of $M$ by Lemma 3.4(1). Since $p \subseteq A n n_{R}\left(A n n_{M}(p)\right)$ and $A n n_{M}(p) \neq 0$, then $A n n_{R}\left(A n n_{M}(p)\right)=p$. Note that there exists a graded completely irreducible submodule $C$ of $M$ such that $A n n_{M}(p) \nsubseteq C$. If not, then $\operatorname{Ann}_{M}(p) \subseteq \bigcap H=0$, where the intersection runs over all graded completely irreducible submodules of $M$, a contradiction. Now, if $A n n_{M}(p) \cap C \neq 0$, then $A n n_{R}\left(A n n_{M}(p) \cap C\right) \neq R$. But $p=A n n_{R}\left(A n n_{M}(p)\right) \subseteq A n n_{R}\left(A n n_{M}(p) \cap C\right)$. So $A n n_{R}\left(A n n_{M}(p) \cap C\right)=$ $p=A n n_{R}\left(A n n_{M}(p)\right)$. Also, it is easy to see that $A n n_{M}(p) \cap C \in \operatorname{Spec}_{G}^{s}(M)$. Since $\phi$ is injective, then $\left|p-\operatorname{Spec}_{G}^{s}(M)\right| \leq 1$ by Proposition 3.2 and thus $A n n_{M}(p)=A n n_{M}(p) \cap C$. This implies that $A n n_{M}(p) \subseteq C$, a contradiction. So we get $A n n_{M}(p) \cap C=0$ and hence $A n n_{M}(p) \cong_{G}\left(C+A n n_{M}(p)\right) / C \subseteq$ $M / C$. By [13, Lemma 2.10], $M / C$ is a graded cocyclic module and thus $\operatorname{Ann}_{M}(p)$ is graded cocyclic by Lemma 3.4(2), (3).

Corollary 3.6. If $M$ is a comultiplication graded $R$-module, then $A n n_{M}(p)$ is a graded cocyclic $R$-module for every $p \in \operatorname{Max}_{G}(R)$ with $A n n_{M}(p) \neq 0$.

Proof. By Corollary 3.5 and Proposition 3.3.

Theorem 3.7. Let $M$ be a $G$-graded $R$-module. If the natural map $\phi$ of $\operatorname{Spec}_{G}^{s}(M)$ is surjective, then $\phi$ is both open and closed; more precisely, for every $N \leq_{G} M, \phi\left(V_{G}^{s}(N)\right)=V_{G}^{\bar{R}}\left(\overline{\operatorname{Ann}_{R}(N)}\right)$ and $\phi\left(\operatorname{Spec}_{G}^{s}(M)-V_{G}^{s}(N)\right)=$ $\operatorname{Spec}_{G}(\bar{R})-V_{G}^{\bar{R}}\left(\overline{\operatorname{Ann}_{R}(N)}\right)$.

Proof. By Proposition 3.1. we have $\phi^{-1}\left(V_{G}^{\bar{R}}(\bar{I})\right)=V_{G}^{s}\left(A n n_{M}(I)\right)$ for every $I \triangleleft_{G} R$ containing $A n n_{R}(M)$ and thus $\phi^{-1}\left(V_{G}^{\bar{R}}\left(\overline{A n n_{R}(N)}\right)\right)=V_{G}^{s}\left(A n n_{M}\left(A n n_{R}(N)\right)\right)=$ $V_{G}^{s}(N)$. So $\phi\left(V_{G}^{s}(N)\right)=V_{G}^{\bar{R}}\left(\overline{A n n_{R}(N)}\right)$ as $\phi$ is surjective. For the second part, note that $\operatorname{Spec}_{G}^{s}(M)-V_{G}^{s}(N)=\operatorname{Spec}_{G}^{s}(M)-\phi^{-1}\left(V_{G}^{\bar{R}}\left(\overline{\operatorname{Ann}_{R}(N)}\right)\right)=$ $\left.\phi^{-1}\left(\operatorname{Spec}_{G}(\bar{R})-V_{G}^{\bar{R}}\left(\overline{A n n_{R}(N)}\right)\right)\right)$. This implies that $\phi\left(\operatorname{Spec}_{G}^{s}(M)-V_{G}^{s}(N)\right)=$ $\operatorname{Spec}_{G}(\bar{R})-V_{G}^{\bar{R}}\left(\overline{A n n_{R}(N)}\right)$. 
Corollary 3.8. Let $M$ be a $G$-graded $R$-module. Then the natural map $\phi$ of $\operatorname{Spec}_{G}^{s}(M)$ is bijective if and only if $\phi$ is homeomorphic.

Let $R$ be a $G$-graded ring and $I \triangleleft_{G} R$. The set $D_{r}=\operatorname{Spec}_{G}(R)-V_{G}^{R}(r R)=$ $\operatorname{Spec}_{G}(R)-V_{G}^{R}(r)$ is open in $\operatorname{Spec}_{G}(R)$ for each $r \in h(R)$ and the family $\left\{D_{r} \mid r \in h(R)\right\}$ forms a base for the Zariski topology on $\operatorname{Spec}_{G}(R)$ (see $[10$, Theorem 2.3]). The radical of $I$, the graded radical of $I$, the nilradical of $I$ and the graded nilradical of $I$ will be denoted by $\sqrt{I}, G r(I), N(R)$ and $N_{G}(R)$, respectively. For details concerning the graded radical and the graded nilradical of a graded ideal of a graded ring, see [11].

Now, we need the following lemma to give a simple characterization for the connectedness of $\operatorname{Spec}_{G}^{s}(M)$.

Lemma 3.9. Let $R$ be a G-graded ring. Then $\operatorname{Spec}_{G}(R)$ is disconnected if and only if $R$ contains idempotent element other than 0 and 1 .

Proof. $\Rightarrow$ : Suppose that $\operatorname{Spec}_{G}(R)$ is disconnected. Then $\operatorname{Spec}_{G}(R)=$ $V_{G}^{R}(I) \cup V_{G}^{R}(J)$ for some $I, J \triangleleft_{G} R$ such that $V_{G}^{R}(I) \cap V_{G}^{R}(J)=\emptyset$ and $V_{G}^{R}(I), V_{G}^{R}(J) \neq \emptyset$. By $\left[10\right.$, Proposition 2.1(2)], $V_{G}^{R}(I+J)=V_{G}^{R}(I) \cap$ $V_{G}^{R}(J)=\emptyset$ and hence $I+J=R$. So there exist $a \in I$ and $b \in J$ such that $a+b=1$ which follows that $1=1_{e}=(a+b)_{e}=a_{e}+b_{e}$ with $a_{e} \in I$ and $b_{e} \in J$. Also by 10, Proposition 2.1(3)], we have $\operatorname{Spec}_{G}(R)=V_{G}^{R}(I J)$. This implies that for every $p \in \operatorname{Spec}_{G}(R)$, we have $I J \subseteq p$ and thus $a_{e} b_{e} \in N_{G}(R)$ by [11, Proposition 2.5]. Since $a_{e} b_{e} \in h(R)$, then $a_{e} b_{e} \in N(R)$ and hence there exists a positive integer $m$ such that $\left(a_{e} b_{e}\right)^{m}=0$. Note that $V_{G}^{R}\left(R a_{e}^{m}+R b_{e}^{m}\right)=\emptyset$. If not, then $\exists p \in \operatorname{Spec}_{G}(R)$ such that $a_{e}^{m}, b_{e}^{m} \in p$ which follows that $a_{e}, b_{e} \in \sqrt{p}$. But $a_{e}, b_{e} \in h(R)$. So we get $a_{e}, b_{e} \in G r(p)=p$ by [11, Proposition 2.4(5)]. Thus $1=a_{e}+b_{e} \in p$, a contradiction. Therefore $R a_{e}^{m}+R b_{e}^{m}=R$ which implies that $1=r a_{e}^{m}+r^{\prime} b_{e}^{m}$ for some $r, r^{\prime} \in R$. Note that $\left(r a_{e}^{m}\right)^{2}=\left(r a_{e}^{m}\right)\left(1-r^{\prime} b_{e}^{m}\right)=r a_{e}^{m}-r r^{\prime}\left(a_{e} b_{e}\right)^{m}=r a_{e}^{m}$. If $r a_{e}^{m}=0$, then $b_{e}^{m} \in U(R)$ and thus $D_{b_{e}^{m}}=\operatorname{Spec}_{G}(R)$ by [11, Proposition 3.6]. So we obtain $V_{G}^{R}\left(R b_{e}^{m}\right)=\emptyset$ and so $V_{G}^{R}(J) \subseteq V_{G}^{R}\left(R b_{e}^{m}\right)=\emptyset$, a contradiction. Similarly, if $r a_{e}^{m}=1$, then we have $V_{G}^{R}(I)=\emptyset$, a contradiction. Therefore, $r a_{e}^{m}$ is an idempotent element other than 0 and 1.

$\Leftarrow$ : Suppose that $x$ is an idempotent element of $R$ other than 0 and 1 . By [15, Lemma 4.4], $x \in R_{e}$ and thus $1-x \in R_{e}$ as $1 \in R_{e}$. By [10, Theorem 2.3(2)], $D_{x} \cap D_{1-x}=D_{x(1-x)}=D_{0}=\emptyset$. Now for any $p \in \operatorname{Spec}_{G}(R)$, we have $x \notin p$ or $1-x \notin p$ which follows that $p \in D_{x} \cup D_{1-x}$ and thus $\operatorname{Spec}_{G}(R)=D_{x} \cup D_{1-x}$. Note that if $D_{x}=\emptyset$, then $x \in N(R)$ by 11, Proposition 3.6(2)]. So $x^{n}=0$ for some positive integer $n$. But $x$ is idempotent which follows that $x=0$, a contradiction. Thus $D_{x} \neq \emptyset$. Similarly, if $D_{1-x}=\emptyset$, then we obtain $x=1$, a contradiction. Hence $D_{1-x} \neq \emptyset$. Now $\operatorname{Spec}_{G}(R)$ can be written as a union two non-empty disjoint open sets which means that $\operatorname{Spec}_{G}(R)$ is disconnected.

The following is a result for Proposition 3.1. Theorem 3.7 and Lemma 3.9 . 
Corollary 3.10. Let $M$ be a G-graded $R$-module such that the natural map $\phi$ of $\operatorname{Spec}_{G}^{s}(M)$ is surjective. Then the following statements are equivalent:

(1) $\operatorname{Spec}_{G}^{s}(M)$ is connected.

(2) $\operatorname{Spec}_{G}(\bar{R})$ is connected.

(3) The only idempotent elements of $\bar{R}$ are $\overline{0}$ and $\overline{1}$.

Proof. $(1) \Rightarrow(2)$ : Since $\phi$ is continuous surjective map and $\operatorname{Spec}_{G}^{s}(M)$ is connected, then $\operatorname{Spec}_{G}(\bar{R})$ is connected.

$(2) \Rightarrow(1)$ : Assume by way of contradiction that $\operatorname{Spec}_{G}^{s}(M)$ is disconnected. Then there exists a clopen set $U$ in $\operatorname{Spec}_{G}^{s}(M)$ such that $U \neq \emptyset$ and $U \neq \operatorname{Spec}_{G}^{s}(M)$. Since $\phi$ is surjective, then $\phi(U)$ is clopen in $\operatorname{Spec}_{G}(\bar{R})$ by Theorem 3.7. This follows that $\phi(U)=\emptyset$ or $\phi(U)=\operatorname{Spec}_{G}(\bar{R})$. Since $U$ is open in $\operatorname{Spec}_{G}^{s}(M)$, then $U=\operatorname{Spec}_{G}^{s}(M)-V_{G}^{s}(N)$ for some $N \leq_{G} M$. Now if $\phi(U)=\operatorname{Spec}_{G}(\bar{R})$, then $\operatorname{Spec}_{G}(\bar{R})=\phi(U)=\operatorname{Spec}_{G}(\bar{R})-V_{G}^{\bar{R}}\left(\overline{A n n_{R}(N)}\right)$ by Theorem 3.7, again. Thus $V_{G}^{\bar{R}}\left(\overline{A n n_{R}(N)}\right)=\emptyset$ which implies that $\emptyset=$ $\phi^{-1}\left(V_{G}^{\bar{R}}\left(\overline{A n n_{R}(N)}\right)\right)=V_{G}^{s}\left(A n n_{M}\left(A n n_{R}(N)\right)\right)=V_{G}^{s}(N)$ by Proposition 3.1 and Lemma 2.17(3). So $U=\operatorname{Spec}_{G}^{s}(M)$, a contradiction. If $\phi(U)=\emptyset$, then $U \subseteq \phi^{-1}(\phi(U))=\emptyset$. It follows that $U=\emptyset$ which is also a contradiction. Therefore $\operatorname{Spec}_{G}^{s}(M)$ is connected.

The equivalence of (2) and (3) follows from Lemma 3.9.

Corollary 3.11. Let $M$ be a $G$-graded $R$-module such that the natural map $\phi$ of $\operatorname{Spec}_{G}^{s}(M)$ is surjective. If either $R$ is a graded local ring or $A n_{R}(M) \in$ $\operatorname{Spec}_{G}(R)$, then both $\operatorname{Spec}_{G}^{s}(M)$ and $\operatorname{Spec}_{G}(\bar{R})$ are connected.

Proof. First, assume that $R$ is a graded local ring. Then the only idempotent elements of $\bar{R}$ are $\overline{0}$ and $\overline{1}$ (see the proof of [15. Lemma 4.4]). Now the result follows from Corollary 3.10. Secondly, assume that $\operatorname{Ann}_{R}(M) \in \operatorname{Spec}_{G}(R)$ and let $r$ be idempotent element of $R$. Then $\bar{r}=\overline{r^{2}}$ and hence $r(r-1) \in$ $A n n_{R}(M)$. Again, by [15, Lemma 4.4], we have $r \in R_{e}$ and thus $r-1 \in R_{e}$.

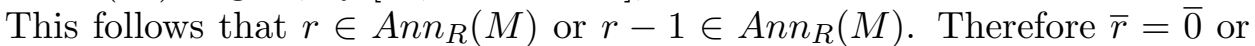
$\bar{r}=\overline{1}$. By Corollary $3.10, \operatorname{Spec}_{G}^{s}(M)$ and $\operatorname{Spec}_{G}(\bar{R})$ are connected.

Proposition 3.12. Let $M$ and $M^{\prime}$ be $G$-graded $R$-modules. If $f: M \rightarrow M^{\prime}$ is a graded module monomorphism, then the mapping $\pi: \operatorname{Spec}_{G}^{s}(M) \rightarrow$ $\operatorname{Spec}_{G}^{s}\left(M^{\prime}\right)$ by $\pi(S)=f(S)$ is continuous.

Proof. It is easy to see that $\pi$ is well-defined. Now for any $S \in \operatorname{Spec}_{G}^{s}(M)$ and any closed set $V_{G}^{S}\left(N^{\prime}\right)$ in $\operatorname{Spec}_{G}^{s}\left(M^{\prime}\right)$, where $N^{\prime} \leq_{G} M^{\prime}$, we have $S \in$ $\pi^{-1}\left(V_{G}^{s}\left(N^{\prime}\right)\right)=\pi^{-1}\left(V_{G}^{s *}\left(A n n_{M^{\prime}}\left(A n n_{R}\left(N^{\prime}\right)\right)\right)\right) \Leftrightarrow \pi(S)=f(S) \subseteq \operatorname{Ann}_{M^{\prime}}\left(A n n_{R}\left(N^{\prime}\right)\right) \Leftrightarrow$ $S=f^{-1}(f(S)) \subseteq f^{-1}\left(A n n_{M^{\prime}}\left(A n n_{R}\left(N^{\prime}\right)\right)\right)=A n n_{M}\left(A n n_{R}\left(N^{\prime}\right)\right) \Leftrightarrow S \in$ $V_{G}^{s *}\left(A n n_{M}\left(A n n_{R}\left(N^{\prime}\right)\right)\right)=V_{G}^{s}\left(A n n_{M}\left(A n n_{R}\left(N^{\prime}\right)\right)\right)$. So $\pi^{-1}\left(V_{G}^{s}\left(N^{\prime}\right)\right)=V_{G}^{s}\left(A n n_{M}\left(A n n_{R}\left(N^{\prime}\right)\right)\right)$ and so $\pi$ is continuous.

Let $M$ be a $G$-graded $R$-module. Now, we write $X_{r}^{s}=\operatorname{Spec}_{G}^{s}(M)-$ $V_{G}^{s}(r M)$ for each $r \in h(R)$ and prove that $B=\left\{X_{r}^{s} \mid r \in h(R)\right\}$ is a base for the Zariski topology on $\operatorname{Spec}_{G}^{s}(M)$, which is similar to that on $\operatorname{Spec}_{G}(R)$. 
Proposition 3.13. For any $G$-graded $R$-module $M$, the set $B=\left\{X_{r}^{s} \mid r \in\right.$ $h(R)\}$ forms a base for the Zariski topology on $\operatorname{Spec}_{G}^{s}(M)$.

Proof. Let $U=\operatorname{Spec}_{G}^{s}(M)-V_{G}^{s}(N)$ be an open set in $\operatorname{Spec}_{G}^{s}(M)$, where $N \leq_{G} M$. Let $S \in U$ and it is enough to find an element $r \in h(R)$ such that $S \in X_{r}^{s} \subseteq U$. Since $S \in U$, then $A n n_{R}(N) \nsubseteq A n n_{R}(S)$ and hence there exists $t \in R$ and $g \in G$ such that $t_{g} \in A n n_{R}(N)-A n n_{R}(S)$. Take $r=t_{g} \in h(R)$. If $S \notin X_{r}^{s}$, then $r R \subseteq A n n_{R}\left(A n n_{M}(r)\right) \subseteq A n n_{R}(S)$ and thus $r \in A n n_{R}(S)$, a contradiction. This implies that $S \in X_{r}^{s}$. Since $r \in$ $A n n_{R}(N)$, then $A n n_{M}\left(A n n_{R}(N)\right) \subseteq A n n_{M}(r)$. Now let $S^{\prime} \in X_{r}^{s}$. Then we have $A n n_{R}\left(A n n_{M}(r)\right) \nsubseteq A n n_{R}\left(S^{\prime}\right)$. If $S^{\prime} \notin U$, then $A n n_{R}(N) \subseteq A n n_{R}\left(S^{\prime}\right)$ which follows that $A n n_{M}\left(A n n_{R}\left(S^{\prime}\right)\right) \subseteq A n n_{M}\left(A n n_{R}(N)\right) \subseteq A n n_{M}(r)$. So $A n n_{R}\left(A n n_{M}(r)\right) \subseteq A n n_{R}\left(A n n_{M}\left(A n n_{R}\left(S^{\prime}\right)\right)\right)=A n n_{R}\left(S^{\prime}\right)$, a contradiction. This means that $S^{\prime} \in U$ and hence $S \in X_{r}^{s} \subseteq U$, as desired.

Proposition 3.14. Let $M$ be a G-graded $R$-module and $r \in h(R)$. Then:

(1) $\phi^{-1}\left(D_{\bar{r}}\right)=X_{r}^{s}$.

(2) $\phi\left(X_{s}^{r}\right) \subseteq D_{\bar{r}}$. If the natural map $\phi$ is surjective, then the equality holds.

(3) $X_{a}^{s} \cap X_{b}^{s}=X_{a b}^{s}$, for any $a, b \in h(R)$.

(4) If $r \in N(R)$, then $X_{r}^{s}=\emptyset$.

(5) If $r \in U(R)$, then $X_{r}^{s}=X_{r^{-1}}^{s}=\operatorname{Spec}_{G}^{s}(M)$.

Proof. (1) $\phi^{-1}\left(D_{\bar{r}}\right)=\phi^{-1}\left(\operatorname{Spec}_{G}(\bar{R})-V_{G}^{\bar{R}}(\bar{r} \bar{R})\right)=\operatorname{Spec}_{G}^{s}(M)-\phi^{-1}\left(V_{G}^{\bar{R}}(\bar{r} \bar{R})\right)=$ $\operatorname{Spec}_{G}^{s}(M)-V_{G}^{s}\left(\operatorname{Ann}_{M}(r)\right)=X_{r}^{s}$ by Proposition 3.1.

(2) Trivial.

(3) By $\left[10\right.$, Theorem 2.3(2)], we have $D_{\bar{a}} \cap D_{\bar{b}}=D_{\overline{a b}}$ and thus $X_{a}^{s} \cap X_{b}^{s}=$ $\phi^{-1}\left(D_{\bar{a}} \cap \phi^{-1}\left(D_{\bar{b}}\right)=\phi^{-1}\left(D_{\overline{a b}}\right)=X_{a b}^{s}\right.$.

(4) Suppose that $r \in N(R)$. By [11, Proposition 3.6(2)], we have $D_{r}=\emptyset$ and hence $D_{\bar{r}}=\emptyset$. This follows that $X_{r}^{s}=\phi^{-1}\left(D_{\bar{r}}\right)=\emptyset$ by $(1)$.

(5) Assume that $r \in U(R)$. Then $D_{r}=\operatorname{Spec}_{G}(R)$ by [11, Proposition 3.6(3)]. This implies that $D_{\bar{r}}=\operatorname{Spec}_{G}(\bar{R})$ and thus $X_{r}^{s}=\phi^{-1}\left(D_{\bar{r}}\right)=$ $\phi^{-1}\left(\operatorname{Spec}_{G}(\bar{R})\right)=\operatorname{Spec}_{G}^{s}(M)$ by $(1)$. Now, since $r \in U(R) \cap h(R)$, then $r^{-1} \in U(R) \cap h(R)$. Therefore $X_{r^{-1}}^{s}=\operatorname{Spec}_{G}^{s}(M)=X_{r}^{s}$.

Let $M$ be a $G$-graded $R$-module. By 10 , Theorem 2.3(4)], each $D_{r}$ is quasi-compact and hence $D_{1}=\operatorname{Spec}_{G}(R)$ is quasi-compact. In the following theorem, we give a similar result for $\operatorname{Spec}_{G}^{s}(M)$ and its basic open sets.

Theorem 3.15. Let $M$ be a G-graded $R$-module. If the natural map $\phi$ is surjective, then the open set $X_{r}^{s}$ is quasi compact for every $r \in h(R)$. Specifically, $\operatorname{Spec}_{G}^{s}(M)$ is quasi compact.

Proof. Let $r \in h(R)$ and $\zeta=\left\{X_{t}^{s} \mid t \in \Delta\right\}$ be a basic open cover for $X_{r}^{s}$, where $\Delta$ is an index set subset of $h(R)$. Then $X_{r}^{s} \subseteq \bigcup_{t \in \Delta} X_{r}^{s}$ and hence $D_{\bar{r}}=$ $\phi\left(X_{r}^{s}\right) \subseteq \bigcup_{t \in \Delta} \phi\left(X_{r}^{s}\right)=\bigcup_{t \in \Delta} D_{\bar{t}}$ by Proposition 3.14(1). So $\bar{\zeta}=\left\{D_{\bar{t}} \mid t \in \Delta\right\}$ 
is a basic open cover for the quasi compact set $D_{\bar{r}}$. Thus it has a finite subcover $\overline{\bar{\zeta}}=\left\{D_{\overline{t_{i}}} \mid i=1, \ldots, n\right\}$, where $t_{i} \in \Delta$ for any $i=1, \ldots, n$. Now $X_{r}^{s}=\phi^{-1}\left(D_{\bar{r}}\right) \subseteq \bigcup_{i=1}^{n} \phi^{-1}\left(D_{\overline{t_{i}}}\right)=\bigcup_{i=1}^{n} X_{\overline{t_{i}}}^{s}$ and this follows that $\underline{\zeta}=\left\{X_{\overline{t_{i}}}^{s} \mid\right.$ $i=1, \ldots, n\} \subseteq \zeta$ is a finite subcover for $X_{r}^{s}$. Therefore $X_{r}^{s}$ is quasi compact. Now $\operatorname{Spec}_{G}^{s}(M)=X_{1}^{s}$. So $\operatorname{Spec}_{G}^{s}(M)$ is quasi compact.

\section{Irreducibility in $\operatorname{Spec}_{G}^{s}(M)$}

Let $M$ be a $G$-graded $R$-module and $Y$ be a subset of $\operatorname{Spec}_{G}^{s}(M)$. We will denote the closure of $Y$ in $\operatorname{Spec}_{G}^{s}(M)$ by $C l(Y)$ and the summation $\sum_{S \in Y} S$ by $T(Y)$. If $Z$ is a subset of $\operatorname{Spec}_{G}(R)$, then the intersection of all members of $Z$ will be expressed by $\xi(Y)$.

Proposition 4.1. Let $M$ be a G-graded $R$-module and $Y \subseteq \operatorname{Spec}_{G}^{s}(M)$. Then $C l(Y)=V_{G}^{s}(T(Y))$. In particular, $Y$ is closed if and only if $V_{G}^{s}(T(Y))=$ $Y$.

Proof. It is easy to see that $Y \subseteq V_{G}^{s}(T(Y))$. Let $V_{G}^{s}(N)$ be any closed set in $\operatorname{Spec}_{G}^{s}(M)$ such that $Y \subseteq V_{G}^{s}(N)$ and it is enough to show that $V_{G}^{s}(T(Y)) \subseteq$ $V_{G}^{s}(N)$. So let $S^{\prime} \in V_{G}^{s}(T(Y))$. Then $A n n_{R}(T(Y)) \subseteq A n n_{R}\left(S^{\prime}\right)$. Note that for any $Q \in Y$, we have $A n n_{R}(N) \subseteq A n n_{R}(Q)$ and thus $A n n_{R}(N) \subseteq$ $\bigcap_{Q \in Y} A n n_{R}(Q)=A n n_{R}(T(Y)) \subseteq A n n_{R}\left(S^{\prime}\right)$. Hence $S^{\prime} \in V_{G}^{s}(N)$. There$Q \in Y$

fore $V_{G}^{s}(T(Y))$ is the smallest closed set containing $Y$ and this means that $C l(Y)=V_{G}^{s}(T(Y))$.

Recall that a topological space $X$ is irreducible if for any decomposition $X=F_{1} \cup F_{2}$ with closed subsets $F_{i}$ of $X$ with $i=1,2$, we have $F_{1}=X$ or $F_{2}=X$. A subset $X^{\prime}$ of $X$ is irreducible if it is an irreducible topological space with the induced topology. An irreducible component of a topological space is a maximal irreducible subset of it. Let $X$ be a topological space. It is easy to see that every singleton subset of $X$ is irreducible. Furthermore, a subset $Y$ of $X$ is irreducible if and only if its closure is irreducible (see $[7$ ).

Theorem 4.2. Let $M$ be a G-graded $R$-module. If $S \in \operatorname{Spec}_{G}^{s}(M)$, then $C l(\{S\})=V_{G}^{s}(S)$ and $V_{G}^{s}(S)$ is an irreducible closed subset of $\operatorname{Spec}_{G}^{s}(M)$.

Proof. By Proposition 4.1, $C l(\{S\})=V_{G}^{s}(T(\{S\}))=V_{G}^{s}(S)$. Since $\{S\}$ is irreducible, then $C l(\{S\})=V_{G}^{s}(S)$ is irreducible, as desired.

Proposition 4.3. Let $M$ be a G-graded $R$-module and $Y \subseteq \operatorname{Spec}_{G}^{s}(M)$. Then :

(1) If $T(Y) \in \operatorname{Spec}_{G}^{s}(M)$, then $Y$ is irreducible.

(2) If $Y$ is irreducible, then $\Upsilon=\left\{A n n_{R}(S) \mid S \in Y\right\}$ is an irreducible subset of $\operatorname{Spec}_{G}(R)$, i.e., $\xi(\Upsilon)=A n n_{R}(T(Y))$ is a graded prime ideal of $R$. 
Proof. (1) Assume that $T(Y) \in \operatorname{Spec}_{G}^{s}(M)$. By Proposition 4.1 and Theorem 4.2, $V_{G}^{s}(T(Y))=C l(Y)$ is irreducible. Therefore $Y$ is irreducible.

(2) Assume that $Y$ is irreducible. Then $\phi(Y)=Y^{\prime}$ is an irreducible subset of $\operatorname{Spec}_{G}(\bar{R})$ since $\phi$ is continuous by Proposition 3.1. Note that $\xi\left(Y^{\prime}\right)=$ $\xi(\phi(Y))=\bigcap_{S \in Y} \overline{A n n_{R}(S)}=\overline{\bigcap_{S \in Y} A n n_{R}(S)}=\overline{A n n_{R}(T(Y))}$ and thus $\xi\left(Y^{\prime}\right)=$ $\overline{A n n_{R}(T(Y))} \in \operatorname{Spec}_{G}(\bar{R})$ by $[14$, Lemma 4.3]. This implies that $\xi(\Upsilon)=$ $A n n_{R}(T(Y)) \in \operatorname{Spec}_{G}(R)$ and thus $\Upsilon$ is an irreducible subset of $\operatorname{Spec}_{G}(R)$ by [14, Lemma 4.3] again.

Corollary 4.4. Let $M$ be a $G$-graded $R$-module. Assume that $p$-Spec ${ }_{G}^{s}(M) \neq$ $\emptyset$ for some $p \in \operatorname{Spec}_{G}(R)$. Then the following hold:

(1) $p-\operatorname{Spec}_{G}^{s}(M)$ is irreducible.

(2) If $p \in \operatorname{Max}_{G}(R)$, then $p$-Spec ${ }_{G}^{s}(M)$ is an irreducible closed subset of $\operatorname{Spec}_{G}^{s}(M)$.

Proof. By [5, Proposition 2.4(a)], we have T(p-Spec $\left.C_{G}^{s}(M)\right) \in p-\operatorname{Spec}_{G}^{s}(M)$. So $p$-Spec ${ }_{G}^{s}(M)$ is irreducible subset of $\operatorname{Spec}_{G}^{s}(M)$ by Proposition 4.3(1). Now it is easy to see that $\operatorname{Ann}_{M}(p) \neq 0$. So if $p \in \operatorname{Max}_{G}(R)$, then $A n n_{R}\left(A n n_{M}(p)\right)=p$. By Lemma 2.17(2), we obtain $V_{G}^{s}\left(A n n_{M}(p)\right)=$ $q_{q \in V_{G}^{R}\left(A n n_{R}\left(A n n_{M}(p)\right)\right)} q-S p e c_{G}^{s}(M)=\bigcup_{q \in V_{G}^{R}(p)} q-S p e c_{G}^{s}(M)=\bigcup_{q \in\{p\}} q-S p e c_{G}^{s}(M)=$ $p$-Spec $G_{G}^{S}(M)$. Therefore $p$-Spec $C_{G}^{s}(M)=V_{G}^{S}\left(A_{n} n_{M}(p)\right)$ and this follows that $p$-Spec $G_{G}^{s}(M)$ is an irreducible closed subset of $\operatorname{Spec}_{G}^{s}(M)$.

Proposition 4.5. Let $M$ be a $G$-graded $R$-module and $Y \subseteq \operatorname{Spec}_{G}^{s}(M)$ such that $\operatorname{Ann}_{R}(T(Y))=p$ is a graded prime ideal of $R$. If $p$-Spec ${ }_{G}^{s}(M) \neq \emptyset$, then $Y$ is irreducible.

Proof. Let $S \in p$-Spec $\operatorname{Sp}_{G}^{s}(M)$. By Lemma 2.17(1) and Proposition 4.1, we have $V_{G}^{s}(S)=V_{G}^{s}(T(Y))=C l(Y)$. So $C l(Y)$ is irreducible by Theorem 4.2 and thus $Y$ is irreducible.

Theorem 4.6. Let $M$ be a G-graded $R$-module and $Y \subseteq \operatorname{Spec}_{G}^{s}(M)$. If the natural map $\phi$ is surjective, then $Y$ is an irreducible closed subset of $\operatorname{Spec}_{G}^{s}(M)$ if and only if $Y=V_{G}^{s}(S)$ for some $S \in \operatorname{Spec}_{G}^{s}(M)$.

Proof. Assume that $Y$ is an irreducible closed subset of $\operatorname{Spec}_{G}^{s}(M)$. Then $A n n_{R}(T(Y)) \in \operatorname{Spec}_{G}(R)$ by Proposition 4.3(2). Thus $\overline{A n n_{R}(T(Y))} \in$ $\operatorname{Spec}_{G}(\bar{R})$. Since $\phi$ is surjective, then $A n n_{R}(S)=A n n_{R}(T(Y))$ for some $S \in$ $\operatorname{Spec}_{G}^{s}(M)$. By Lemma 2.17(1) and Proposition 4.1. $V_{G}^{s}(S)=V_{G}^{s}(T(Y))=$ $C l(Y)=Y$. Conversely, if $Y=V_{G}^{s}(S)$ for some $S \in \operatorname{Spec}_{G}^{s}(M)$, then $Y$ is closed and it is irreducible by Theorem 4.2 .

Corollary 4.7. Let $M$ be a $G$-graded $R$-module and $S \in \operatorname{Spec}_{G}^{s}(M)$. If $\overline{A n n_{R}(S)}$ is a minimal graded prime ideal of $\bar{R}$, then $V_{G}^{s}(S)$ is an irreducible component of $\operatorname{Spec}_{G}^{s}(M)$. The converse is true if the natural map $\phi$ is surjective. 
Proof. By Theorem 4.2, $V_{G}^{s}(S)$ is irreducible and it is enough to show that it is a maximal irreducible. Let $Y$ be irreducible subset of $\operatorname{Spec}_{G}^{s}(M)$ with $V_{G}^{s}(S) \subseteq Y$ and it remains to prove $Y=V_{G}^{s}(S)$. Since $S \in V_{G}^{s}(S) \subseteq Y$, then $S \in Y$ and thus $\overline{A n n_{R}(T(Y))} \subseteq \overline{A n n_{R}(S)}$. By Proposition 4.3(2), $\overline{A n n_{R}(T(Y))} \in \operatorname{Spec}_{G}(\bar{R})$. But $\overline{A n n_{R}(S)}$ is a minimal graded prime ideal of $\bar{R}$. So $\overline{A n n_{R}(T(Y))}=\overline{A n n_{R}(S)}$ and this follows that $V_{G}^{\bar{R}}\left(\overline{A n n_{R}(T(Y))}\right)=$ $V_{G}^{\bar{R}}\left(\overline{A n n_{R}(S)}\right)$. Thus $V_{G}^{s}(T(Y))=\phi^{-1}\left(V_{G}^{\bar{R}}\left(\overline{A n n_{R}(T(Y))}\right)=\phi^{-1}\left(V_{G}^{\bar{R}}\left(\overline{A n n_{R}(S)}\right)\right)=\right.$ $V_{G}^{s}(S)$ by Lemma 2.17(3) and Proposition 3.1. Since $Y \subseteq V_{G}^{s}(T(Y))$, then $Y \subseteq V_{G}^{s}(S)$ and hence $Y=V_{G}^{s}(S)$. Conversely, assume that $\phi$ is surjective. Let $\bar{p} \in \operatorname{Spec}_{G}(\bar{R})$ with $\bar{p} \subseteq \overline{A n n_{R}(S)}$ and it is enough to show that $\bar{p}=$ $\overline{A n n_{R}(S)}$. By the surjectivity of $\phi$, there exists $S^{\prime} \in S p e c_{G}^{s}(M)$ such that $A n n_{R}\left(S^{\prime}\right)=p$. Then $A n n_{R}\left(S^{\prime}\right) \subseteq A n n_{R}(S)$. So we get $V_{G}^{s}(S) \subseteq V_{G}^{s}\left(S^{\prime}\right)$. Since $V_{G}^{s}(S)$ is irreducible component and $V_{G}^{s}\left(S^{\prime}\right)$ is irreducible by Theorem 4.2, then $V_{G}^{s}(S)=V_{G}^{s}\left(S^{\prime}\right)$. By Lemma 2.17(1), we have $A n n_{R}(S)=$ $A n n_{R}\left(S^{\prime}\right)=p$. Therefore $\bar{p}=\overline{A n n_{R}(S)}$, as needed.

Theorem 4.8. Let $M$ be a $G$-graded $R$-module. Then the following statements are equivalent for any $S_{1}, S_{2} \in \operatorname{Spec}_{G}^{s}(M)$ :

(1) $\operatorname{Spec}_{G}^{s}(M)$ is a $T_{0}$-space.

(2) If $V_{G}^{s}\left(S_{1}\right)=V_{G}^{s}\left(S_{2}\right)$, then $S_{1}=S_{2}$.

(3) $\left|p-\operatorname{Spec}_{G}^{s}(M)\right| \leq 1$, for every $p \in \operatorname{Spec}_{G}(R)$.

(4) The natural map $\phi$ is injective.

Proof. The equivalence of (2), (3) and (4) follows from Proposition 3.2. Also $(1) \Leftrightarrow(2)$ follows from Theorem 4.2 and the fact that a topological space is a $T_{0}$-space if the closures of distinct points are distinct.

Proposition 4.9. Let $M$ be a $G$-graded module over a graded field $F$. If $\operatorname{Spec}_{G}^{s}(M)$ is a $T_{0}$-space, then $|A| \leq 1$ for any base $A$ of $M$ with $A \subseteq h(M)$.

Proof. Suppose that there exists a base $A$ of $M$ such that $A \subseteq h(M)$ and $|A|>1$. By Lemma 2.8(1),(2), there exist $N_{1}, N_{2} \in \operatorname{Spec}_{G}^{s}(M)$ with $N_{1} \neq$ $N_{2}$. Since $F$ is a $G$-graded field, then $r \in U(F)$ for every $r \in h(F)-\{0\}$ and hence $B=\left\{X_{r} \mid r \in h(F)\right\}=\left\{\operatorname{Spec}_{G}^{s}(M), \emptyset\right\}$ by Proposition 3.14(5). This follows that $\tau_{G}^{s}=$ the trivial topology on $\operatorname{Spec}_{G}^{s}(M)$. Now it is easy to see that $\operatorname{Spec}_{G}^{s}(M)$ is not a $T_{0}$-space, a contradiction.

Recall that a topological space $X$ is called a $T_{1}$-space if every singleton subset of $X$ is closed. Now, we need the following lemma to investigate $\operatorname{Spec}_{G}^{s}(M)$ with the Zariski topology from the viewpoint of being a $T_{1}$-space.

Lemma 4.10. Let $M$ be a $G$-graded $R$-module. If $Q$ is a graded minimal submodule of $M$, then $\operatorname{Ann}_{R}(Q)$ is a graded maximal ideal of $R$.

Proof. Since $Q \neq 0$, then $A n n_{R}(Q) \neq R$. Let $J \triangleleft_{G} R$ such that $A n n_{R}(Q) \subseteq$ $J \subseteq R$ and $A n n_{R}(Q) \neq J$. Then $\exists t \in J-A n n_{R}(Q)$. So $\exists m \in h(M) \cap Q$ such that $t m \neq 0$. Note that $J m$ is a non-zero graded submodule of $M$. But $J m \subseteq Q$. So $J m=Q$ as $Q$ is a graded minimal submodule. Thus $m=j m$ 
for some $j \in J$. This implies that $1-j \in A n n_{R}(m) \subseteq A n n_{R}(J m)=$ $A n n_{R}(Q) \subseteq J$. Hence $1 \in J$. Therefore $J=R$.

Theorem 4.11. Let $M$ be a $G$-graded $R$-module such that every graded second submodule of $M$ contains a graded minimal submodule and let $S \in$ $\operatorname{Spec}_{G}^{s}(M)$. Then the set $\{S\}$ is closed in $\operatorname{Spec}_{G}^{s}(M)$ if and only if $S$ is a graded minimal submodule of $M$ and $p$-Spec ${ }_{G}^{s}(M)=\{S\}$, where $p=$ $A n n_{R}(S)$.

Proof. Suppose that $\{S\}$ is closed in $\operatorname{Spec}_{G}^{s}(M)$. Then $C l(\{S\})=\{S\}$ and hence $V_{G}^{s}(S)=\{S\}$ by Theorem 4.2. Now $\exists W \in \operatorname{Min}_{G}(M)$ such that $W \subseteq S$. So we get $A n n_{R}(S) \subseteq A n n_{R}(W)$, which implies that $W \in$ $V_{G}^{s}(S)=\{S\}$. Hence $W=S$ and this means that $S \in \operatorname{Min}_{G}(M)$. Also it is clear that $p-\operatorname{Spec}_{G}^{s}(M)=\{S\}$. Conversely, let $H \in C l(\{S\})$. Then $H \in V_{G}^{s}(S)$ and hence $A n n_{R}(S) \subseteq A n n_{R}(H)$. By Lemma 4.10, we have $A n n_{R}(S) \in \operatorname{Max}_{G}(R)$. This follows that $A n n_{R}(S)=A n n_{R}(H)$. Since $p$-Spec ${ }_{G}^{s}(M)=\{S\}$, then $H=S$. Therefore $C l(\{S\})=\{S\}$, as desired.

Corollary 4.12. Let $M$ be a G-graded $R$-module such that every graded second submodule contains a graded minimal submodule. Then $\operatorname{Spec}_{G}^{s}(M)$ is a $T_{1}$-space if and only if $\operatorname{Min}_{G}(M)=\operatorname{Spec}_{G}^{S}(M)$.

Proof. Assume that $\operatorname{Spec}_{G}(M)$ is a $T_{1}$-space. By [5, Proposition 2.3(c)], $\operatorname{Min}_{G}(M) \subseteq \operatorname{Spec}_{G}^{s}(M)$. Let $S \in \operatorname{Spec}_{G}^{s}(M)$. Since $\operatorname{Spec}_{G}^{s}(M)$ is a $T_{1}$-space, then $\{S\}$ is closed in $\operatorname{Spec}_{G}^{s}(M)$. By Theorem 4.11, we have $S \in \operatorname{Min}_{G}(M)$. For the converse, assume that $\operatorname{Min}_{G}(M)=\operatorname{Spec}_{G}^{s}(M)$. Let $W \in \operatorname{Spec}_{G}^{s}(M)$ and it is enough to show that $\{W\}$ is closed in $\operatorname{Spec}_{G}^{s}(M)$. Let $p=A n n_{R}(W)$ and $H \in p$-Spec $G_{G}^{s}(M)$. By [5, Proposition 2.4(a)], we have $H+W \in p$ $\operatorname{Spec}_{G}^{s}(M)$. This follows that $H+W \in \operatorname{Min}_{G}(M)$. But $H, W \subseteq H+W$. So $H=W$ and so $p-\operatorname{Spec}_{G}^{s}(M)=\{W\}$. By Theorem 4.11, $\{W\}$ is closed in $\operatorname{Spec}_{G}^{s}(M)$.

Let $M$ be a $G$-graded $R$-module and $S \in \operatorname{Spec}_{G}^{s}(M)$. We say that $S$ is a maximal graded second submodule if it is not properly contained in another graded second submodule of $M$ (see [8]).

Proposition 4.13. Let $M$ be a G-graded $R$-module. Then the following hold:

(1) If $\operatorname{Spec}_{G}^{s}(M)$ is a $T_{1}$-space, then every graded second submodule of $M$ is a maximal graded second.

(2) If $M$ is a graded Artinian module, then $\operatorname{Spec}_{G}^{s}(M)$ is a $T_{1}$-space if and only if $\operatorname{Spec}_{G}^{s}(M)$ is discrete.

Proof. (1) Let $S \in \operatorname{Spec}_{G}^{s}(M)$ and we show that it is maximal graded second. So let $S^{\prime} \in \operatorname{Spec}_{G}^{s}(M)$ with $S \subseteq S^{\prime}$. By hypothesis, $\{S\}=C l(\{S\}) \subseteq$ $C l\left(\left\{S^{\prime}\right\}\right)=\left\{S^{\prime}\right\}$ and thus $S=S^{\prime}$.

(2) If $M=0$, then the result is trivially true. Suppose that $M \neq 0$. Clearly, if $\operatorname{Spec}_{G}^{s}(M)$ is discrete, then it is a $T_{1}$-space. Conversely, assume that 
$\operatorname{Spec}_{G}^{S}(M)$ is a $T_{1}$-space. Let $\operatorname{Max}_{G}^{S}(M)$ be the set of all maximal graded second submodules of $M$. By (1), $\operatorname{Spec}_{G}^{s}(M) \subseteq \operatorname{Max}_{G}^{s}(M)$. But by [8, Theorem 2.11], $\operatorname{Max}_{G}^{s}(M)$ is a finite set and hence $\operatorname{Spec}_{G}^{s}(M)$ is finite. Now, since $\operatorname{Spec}_{G}^{s}(M)$ is a $T_{1}$-space, then it is discrete with respect to the Zariski topology.

\section{REFERENCES}

[1] K. Al-Zoubi and M and Al-Azaizeh, On graded 2-absorbing second submodules of graded modules over graded commutative rings, Kragujevac Journal of Mathematics 48 (1) (2024), 55-66.

[2] K. Al-Zoubi and A. Al-Qderat, Some properties of graded comultiplication modules, Open Math., 15 (1) (2017), 187-192.

[3] K. Al-Zoubi and M. Jaradat, The Zariski Topology on the graded classical prime spectrum of a graded module over a graded commutative ring, Mat. Vesnik, 70 (2018), 303-313.

[4] K. Al-Zoubi and M. Jaradat,The Zariski topology on the graded primary spectrum over graded commutative rings, Tatra Mt. Math. Publ. 74 (1) (2019), 7-16.

[5] H. Ansari-Toroghy and F.Farshadifar, On graded second modules, Tamkang J. Math., 43 (4) (2012), 499-505

[6] H. Ansari-Toroghy and F. Farshadifar, Graded comultiplication modules, Chiang Mai J. Sci., 38 (3) (2011), 311-320.

[7] N. Bourbaki, Commutative Algebra. Chapter 1-7. Springer-Verlag, Berlin (1989).

[8] S. Çeken and M. Alkan, On graded second and coprimary modules and graded secondary representations, Bull. Malays. Math. Sci. Soc., 38 (4) (2015), 1317-1330.

[9] C. Nastasescu and F. Van Oystaeyen, Methods of Graded Rings, LNM 1836. BerlinHeidelberg: Springer-Verlag, 2004.

[10] N. A. Ozkirisci , K.H. Oral, U. Tekir, Graded prime spectrum of a graded module, Iran. J. Sci.Technol., 37 A3 (2013), 411-420.

[11] M. Refai, M. Hailat and S. Obiedat, Graded radicals on graded prime spectra, Far East J. of Math. Sci., part I (2000), 59-73.

[12] M. Refai, On properties of G-spec(R), Sci. Math. Jpn. 53 (3) (2001), 411-415.

[13] M. Refai and R.Abu-Dawwas, On generalizations of graded second submodules, Proyecciones (Antofagasta) 39 (6) (2020), 1537-1554.

[14] S. Salam and K. Al-Zoubi, The Zariski topology on the graded primary spectrum of a graded module over a graded commutative ring. (submitted), arXiv preprint arXiv:2109.10113 (2021).

[15] F. Van Oystaeyen, Methods in ring theory. Vol. 129. Springer Science and Business Media, 2012

Saif Salam, Department of Mathematics and Statistics, Jordan University of Science and Technology, P.O.Box 3030, Irbid 22110, Jordan.

Email address: smsalam19@sci.just.edu.jo

Khaldoun Al-Zoubi, Department of Mathematics and Statistics, Jordan University of Science and Technology, P.O.Box 3030, Irbid 22110, Jordan.

Email address: kfzoubi@just.edu.jo 